\title{
STABILIZATION OF THE WAVE EQUATION ON 1-D NETWORKS
}

\author{
JULIE VALEIN * AND ENRIQUE ZUAZUA ${ }^{\dagger}$
}

\begin{abstract}
In this paper we study the stabilization of the wave equation on general 1-d networks. For that, we transfer known observability results in the context of control of conservative systems (see [14]) into a weighted observability estimate for the dissipative one. Then we use an interpolation inequality similar to the one proved in [7] to obtain the explicit decay estimates of the energy for smooth initial data. The obtained decay rate depends on the geometric and topological properties of the network. We give also some examples of particular networks in which our results apply yielding different decay rates.
\end{abstract}

Key words. stabilization, wave equation, network

AMS subject classifications. 93D15, 93B07, 35L05

1. Introduction and main results. In this paper, we consider a planar network of elastic strings that undergoes small perpendicular vibrations. Recently, the control, observation and stabilization problems of these networks have been the object of intensive research (see $[14,16]$ and the references therein).

Here we are interested in the problem of stabilization of the network by means of a damping term located on one single exterior node. The aim of this paper is to develop a systematic method to address this issue and to give a general result allowing to transform an observability result for the corresponding conservative system into a stabilization one for the damped one.

Before going on, let us recall some definitions and notations about $1-d$ networks used in the paper. We refer to $[1,19,24]$ for more details.

A $1-d$ network $\mathcal{R}$ is a connected set of $\mathbb{R}^{n}, n \geq 1$, defined by

$$
\mathcal{R}=\bigcup_{j=1}^{N} e_{j}
$$

where $e_{j}$ is a curve that we identify with the interval $\left(0, l_{j}\right), l_{j}>0$, and such that for $k \neq j, \overline{e_{j}} \cap \overline{e_{k}}$ is either empty or a common end called a vertex or a node (here $\overline{e_{j}}$ stands for the closure of $e_{j}$ ).

For a function $u: \mathcal{R} \longrightarrow \mathbb{R}$, we set $u_{j}=u_{\mid e_{j}}$ the restriction of $u$ to the edge $e_{j}$.

We denote by $\mathcal{E}=\left\{e_{j} ; 1 \leq j \leq N\right\}$ the set of edges of $\mathcal{R}$ and by $\mathcal{V}$ the set of vertices of $\mathcal{R}$. For a fixed vertex $v$, let

$$
\mathcal{E}_{v}=\left\{j \in\{1, \ldots, N\} ; v \in \overline{e_{j}}\right\}
$$

be the set of edges having $v$ as vertex. If card $\left(\mathcal{E}_{v}\right)=1, v$ is an exterior node, while if card $\left(\mathcal{E}_{v}\right) \geq 2$, $v$ is an interior one. We denote by $\mathcal{V}_{\text {ext }}$ the set of exterior nodes and by $\mathcal{V}_{\text {int }}$ the set of interior ones. For $v \in \mathcal{V}_{\text {ext }}$, the single element of $\mathcal{E}_{v}$ is denoted by $j_{v}$.

\footnotetext{
*Université de Valenciennes et du Hainaut Cambrésis, LAMAV, FR CNRS 2956, Institut des Sciences et Techniques of Valenciennes, F-59313 - Valenciennes Cedex 9 France, julie.valein@univ-valenciennes.fr, Tel: +33 (0)327 5119 01, Fax: +33 (0)32751 1900 . This work started while the first author was visiting the Universidad Autónoma de Madrid during six months partially supported by a grant 'bourse régionale de mobilité à l'internationale' of the 'Région Nord-Pas de Calais' (France).

†Basque Center for Applied Mathematics (BCAM), Bizkaia Technology Park, E-48170 Zamudio - Basque Country- Spain, zuazua@bcamath.org, Tel: +34 946567 842, Fax: +34946567 843.The work of the second author was supported by the Grant MTM2008-03541 of the MICINN (Spain).
} 
We now fix a partition of $\mathcal{V}_{\text {ext }}: \mathcal{V}_{\text {ext }}=\mathcal{D} \cup\left\{v_{1}\right\}$, where $\mathcal{D} \neq \emptyset$. In this way, we distinguish the conservative exterior nodes, $\mathcal{D}$, in which we impose Dirichlet homogeneous boundary condition, and the one in which the damping term is effective, $v_{1}$. To simplify the notation, we will assume that $v_{1}$ is located at the end 0 of the edge $e_{1}$.

Let $u_{j}=u_{j}(t, x): \mathbb{R} \times\left[0, l_{j}\right] \rightarrow \mathbb{R}$ be the function describing the transversal displacement in time $t$ of the string $e_{j}$ of length $l_{j}$. Let us denote by $L$ the sum of the lengths of all edges of the network, the total length of the network.

We assume that the displacements $u_{j}$ satisfy the following system

$$
\begin{cases}\frac{\partial^{2} u_{j}}{\partial t^{2}}(x, t)-\frac{\partial^{2} u_{j}}{\partial x^{2}}(x, t)=0 & 0<x<l_{j}, t>0, \forall j \in\{1, \ldots, N\}, \\ u_{j}(v, t)=u_{l}(v, t) & \forall j, l \in \mathcal{E}_{v}, v \in \mathcal{V}_{i n t}, t>0, \\ \sum_{j \in \mathcal{E}_{v}} \frac{\partial u_{j}}{\partial n_{j}}(v, t)=0 & \forall v \in \mathcal{V}_{i n t}, t>0, \\ u_{j_{v}}(v, t)=0 & \forall v \in \mathcal{D}, t>0, \\ \frac{\partial u_{1}}{\partial x}(0, t)=\frac{\partial u_{1}}{\partial t}(0, t) & \forall t>0, \\ u(t=0)=u^{(0)}, \frac{\partial u}{\partial t}(t=0)=u^{(1)}, & \end{cases}
$$

where $\partial u_{j} / \partial n_{j}(v,$.$) stands for the outward normal (space) derivative of u_{j}$ at the vertex $v$. We denote by $u$ the vector $u=\left(u_{j}\right)_{j=1, \ldots, N}$.

The above system has been considered by several authors in some particular situations. We refer, for instance, to [6], [2], [3, 4], [5] and [25], where explicit decay rates are obtained for networks with some special structures. We also refer to [20] where the problem is considered in the presence of delay terms in the feedback law.

The object of this paper is not to give an additional result in a particular case, but rather to develop a systematic method allowing to address the issue in a general context. We do this transfering known observability results for the corresponding conservative system into stabilization results for the dissipative one. This provides a new proof for the existing results mentioned above and allows getting new ones.

As mentioned above, in this paper, we consider the case where the dissipation is located on an external node of the network, but the method can be adapted to treat the case where the damping term is located in several nodes, both exterior and interior ones.

In order to study system (1.1) we need a proper functional setting. We define the real Hilbert spaces

$$
L^{2}(\mathcal{R})=\left\{u: \mathcal{R} \rightarrow \mathbb{R} ; u_{j} \in L^{2}\left(0, l_{j}\right), \forall j=1, \cdots, N\right\}
$$

and

$$
V:=\left\{\phi \in \prod_{j=1}^{N} H^{1}\left(0, l_{j}\right): \phi_{j}(v)=\phi_{k}(v) \forall j, k \in \mathcal{E}_{v}, \forall v \in \mathcal{V}_{i n t} ; \phi_{j_{v}}(v)=0 \forall v \in \mathcal{D}\right\},
$$

equipped with the natural inner products

$$
<\phi, \tilde{\phi}>_{L^{2}(\mathcal{R})}=\sum_{j=1}^{N} \int_{0}^{l_{j}} \phi_{j} \tilde{\phi}_{j} d x \text { and }<\phi, \tilde{\phi}>_{V}=\sum_{j=1}^{N} \int_{0}^{l_{j}} \frac{\partial \phi_{j}}{\partial x} \frac{\partial \tilde{\phi}_{j}}{\partial x} d x
$$

respectively. 
It is well known that system (1.1) may be rewritten as the first order evolution equation

$$
\left\{\begin{array}{l}
U^{\prime}=\mathcal{A} U \\
U(0)=\left(u^{(0)}, u^{(1)}\right)
\end{array}\right.
$$

in the Hilbert space

$$
H:=V \times L^{2}(\mathcal{R})
$$

equipped with the usual inner product

$$
\left\langle\left(\begin{array}{c}
u \\
w
\end{array}\right),\left(\begin{array}{c}
\tilde{u} \\
\tilde{w}
\end{array}\right)\right\rangle:=\sum_{j=1}^{N} \int_{0}^{l_{j}}\left(\frac{\partial u_{j}}{\partial x} \frac{\partial \tilde{u}_{j}}{\partial x}+w_{j} \tilde{w}_{j}\right) d x .
$$

Here $U$ is the vector $U=\left(u, \frac{\partial u}{\partial t}\right)^{T}$, the operator $\mathcal{A}$ is defined by

$$
\mathcal{A}\left(\begin{array}{c}
u \\
w
\end{array}\right):=\left(\begin{array}{c}
w \\
\Delta u
\end{array}\right)
$$

$\Delta$ being the Laplace operator: $\Delta u=\left(\frac{\partial^{2} u_{j}}{\partial x^{2}}\right)_{j=1, \ldots, N}$. The domain $D(\mathcal{A})$ of the operator $\mathcal{A}$ is defined by

$$
D(\mathcal{A}):=\left\{(u, w) \in\left(V \cap \prod_{j=1}^{N} H^{2}\left(0, l_{j}\right)\right) \times V: \frac{\partial u_{1}}{\partial x}(0)=w_{1}(0) ; \sum_{j \in \mathcal{E}_{v}} \frac{\partial u_{j}}{\partial n_{j}}(v)=0, \forall v \in \mathcal{V}_{i n t}\right\} .
$$

Since $\mathcal{A}$ is maximal dissipative, by Lumer-Phillips' theorem, for an initial datum $U_{0} \in H$, there exists a unique solution $U \in C([0,+\infty), H)$ to problem (1.2). Moreover, if $U_{0} \in D(\mathcal{A})$, then

$$
U \in C([0,+\infty), D(\mathcal{A})) \cap C^{1}([0,+\infty), H) .
$$

We define the natural energy of $u$ by

$$
E_{u}(t):=\frac{1}{2} \sum_{j=1}^{N} \int_{0}^{l_{j}}\left(\left(\frac{\partial u_{j}}{\partial t}\right)^{2}+\left(\frac{\partial u_{j}}{\partial x}\right)^{2}\right) d x
$$

This energy satisfies

$$
E_{u}^{\prime}(t)=-\left(\frac{\partial u_{1}}{\partial t}(0, t)\right)^{2} \leq 0
$$

and therefore it is decreasing.

REMARK 1.1. Integrating the expression (1.4) between 0 and $T$, we obtain

$$
\int_{0}^{T}\left(\frac{\partial u_{1}}{\partial t}(0, t)\right)^{2} d t=E_{u}(0)-E_{u}(T) \leq E_{u}(0)
$$

Consequently, this estimate implies that $\frac{\partial u_{1}}{\partial t}(0,$.$) belongs to L^{2}(0, T)$ for finite energy solutions. This is a "hidden" regularity property in the sense that it is not a direct consequence of the regularity of finite energy solutions. 
In $[2,3,4,5,6,20]$, the method to obtain the stabilization of (1.1) in particular cases is based on the use of observability estimates for the solutions $\phi$ of the conservative problem without damping, with Neumann boundary condition at the node $x=0$ :

$$
\begin{cases}\frac{\partial^{2} \phi_{j}}{\partial t^{2}}-\frac{\partial^{2} \phi_{j}}{\partial x^{2}}=0 & 0<x<l_{j}, t>0, \forall j \in\{1, \ldots, N\}, \\ \phi_{j}(v, t)=\phi_{l}(v, t) & \forall j, l \in \mathcal{E}_{v}, v \in \mathcal{V}_{i n t}, t>0, \\ \sum_{j \in \mathcal{E}_{v}} \frac{\partial \phi_{j}}{\partial n_{j}}(v, t)=0 & \forall v \in \mathcal{V}_{i n t}, t>0, \\ \phi_{j_{v}}(v, t)=0 & \forall v \in \mathcal{D}, t>0, \\ \frac{\partial \phi_{1}}{\partial x}(0, t)=0 & \forall t>0, \\ \phi(t=0)=u^{(0)}, \frac{\partial \phi}{\partial t}(t=0)=u^{(1)} . & \end{cases}
$$

It is well known that this problem is well posed in the natural energy space. If we suppose that $\left(u^{(0)}, u^{(1)}\right) \in H$, then problem (1.5) admits a unique solution

$$
\phi \in C(0, T ; V) \cap C^{1}\left(0, T ; L^{2}(\mathcal{R})\right) .
$$

This system is obviously conservative and its energy is constant: $E_{\phi}(t)=E_{\phi}(0)$ for all $t>0$. Let us then denote by $\left(\lambda_{n}^{2}\right)_{n \geq 1}$ the sequence of eigenvalues of (1.5) and let $\left(\varphi_{n}\right)_{n \geq 1}$ be the corresponding eigenvectors forming an orthonormal basis of $L^{2}(\mathcal{R})$. In particular cases, in $[2,3,4,5,6,20]$, under appropriate conditions, observability inequalities of the form

$$
\sum_{n \geq 1} c_{n}^{2}\left(\lambda_{n}^{2} a_{n}^{2}+b_{n}^{2}\right) \leq C \int_{0}^{T}\left|\frac{\partial \phi_{1}}{\partial t}(0, t)\right|^{2} d t
$$

are proved for system (1.5), where $a_{n}, b_{n}$ are the Fourier coefficients of the initial data of $\phi$ in the basis $\left(\varphi_{n}\right)_{n}$, and with weights $c_{n}^{2}>0$ depending on the network. To obtain the stabilization result from the observability inequality (1.6), the authors decompose the solution $u$ as the sum of $\phi$, solution of (1.5) with the same initial data than $u$, and a rest with vanishing initial data. Then they show regularity results of trace type for this rest (see section 4 of [20]). However, in these articles, the analysis is limited to strong observability inequalities (leading to exponential or polynomial decay results) which hold only for a restricted class of networks.

In the present paper we are able to deal with arbitrary networks in which weaker observability inequalities may hold leading to arbitrarily slow decay rates.

Our analysis is based on the existing observability results for the following system with Dirichlet boundary conditions at all exterior nodes

$$
\begin{cases}\frac{\partial^{2} \psi_{j}}{\partial t^{2}}(x, t)-\frac{\partial^{2} \psi_{j}}{\partial x^{2}}(x, t)=0 & 0<x<l_{j}, t>0, \forall j \in\{1, \ldots, N\}, \\ \psi_{j}(v, t)=\psi_{l}(v, t) & \forall j, l \in \mathcal{E}_{v}, v \in \mathcal{V}_{\text {int }}, t>0, \\ \sum_{j \in \mathcal{E}_{v}} \frac{\partial \psi_{j}}{\partial n_{j}}(v, t)=0 & \forall v \in \mathcal{V}_{i n t}, t>0, \\ \psi_{j_{v}}(v, t)=0 & \forall v \in \mathcal{D}, t>0 \\ \psi_{1}(0, t)=0 & t>0, \\ \psi(t=0)=\psi^{(0)}, \frac{\partial \psi}{\partial t}(t=0)=\psi^{(1)} . & \end{cases}
$$

The study of the observability of this system is motivated by control problems (see $[9,10,11,12$, $13,14])$.

The difference between systems (1.5) and (1.7) is the boundary condition at the end 0 of $e_{1}$ : the Dirichlet one for (1.7) and Neumann one for (1.5). 
If we suppose that $\left(\psi^{(0)}, \psi^{(1)}\right) \in \tilde{H}=\tilde{V} \times L^{2}(\mathcal{R})$ where

$$
\tilde{V}=\left\{\psi \in \prod_{j=1}^{N} H^{1}\left(0, l_{j}\right): \psi_{j}(v)=\psi_{k}(v) \forall j, k \in \mathcal{E}_{v}, \forall v \in \mathcal{V}_{i n t} ; \psi_{j_{v}}(v)=0, \forall v \in \mathcal{D} ; \psi_{1}(0)=0\right\},
$$

then problem (1.7) admits a unique solution $\psi \in C(0, T ; \tilde{V}) \cap C^{1}\left(0, T ; L^{2}(\mathcal{R})\right)$. This system is obviously also conservative.

In this paper, we show the link between the existing observability results for (1.7) and the stabilization properties of (1.1).

To be more precise, let us denote by $\left(\tilde{\lambda}_{n}^{2}\right)_{n \geq 1}$ the sequence of eigenvalues corresponding to problem (1.7) and let $\left(\varphi_{n}^{D}\right)_{n \geq 1}$ be the corresponding eigenvectors forming an orthonormal basis of $L^{2}(\mathcal{R})$. Under some conditions on the topology of the network and the lengths of the strings entering in it, Dager and Zuazua $[9,10,11,12,13,14]$ proved weighted observability inequalities for (1.7) of the following form

$$
E_{*}^{D}(\psi, 0):=\sum_{n \geq 1} c_{n}^{2}\left(\tilde{\lambda}_{n}^{2} \psi_{0, n}^{2}+\psi_{1, n}^{2}\right) \leq C \int_{0}^{T}\left|\frac{\partial \psi_{1}}{\partial x}(0, t)\right|^{2} d t
$$

for a positive constant $C$, where $\psi_{0, n}, \psi_{1, n}$ are the Fourier coefficients of the initial data of $\psi$ in the basis $\left(\varphi_{n}^{D}\right)_{n}$ and with positive weights $\left(c_{n}^{2}\right)_{n \geq 1}$ depending on the properties of the network.

To obtain this weighted observability estimate (1.8), several methods have been developed in those articles. The first one, that uses the D'Alembert representation formula, applies to treeshaped networks. Its main advantage is that it does not require computing the spectrum of the network. This method has been later adapted in [3] and [5] to analyze the stabilization of stars and trees. A second method uses the Fourier expansion of solutions and the Beurling-Malliavin Theorem (see for instance [13, 14, 20]). This applies for general networks and avoids the difficulties related to applying the D'Alembert formula to more complex networks that may contain circuits (see, for instance, [?]).

The key observation of this paper is that the weighted observability estimates for $u$ solution of (1.1) can be obtained directly from (1.8). This method is of systematic application and avoids remaking all the fine analysis already carried out in [9, 14], that uses, in particular, tools from Number Theory, to analyze the properties of the weights $\left\{c_{n}^{2}\right\}_{n \geq 1}$ in terms of those of the network under consideration.

Let us now explain how (1.8) can be applied directly in our context. For that we decompose $u$ as the sum of $w$, a solution of (1.7) with appropriate initial data $\left(u^{(0)}-u_{1}^{(0)}(0) \varphi, u^{(1)}\right)$ (where $\varphi$ is a given smooth function such that $\varphi_{1}(0)=1$ ), and a rest. Applying (1.8) to the solution $w$ of (1.7), we obtain the following weighted observability estimate for $u$ solution of (1.1)

$$
E_{*}^{D}(w, 0)+u_{1}^{(0)}(0)^{2} \leq C_{T} \int_{0}^{T}\left(\frac{\partial u_{1}}{\partial t}(0, t)\right)^{2} d t
$$

where $E_{*}^{D}(w, 0)$ is defined by $(1.8)$, with weights $\left(c_{n}^{2}\right)_{n}$ depending on the network. If the weights $c_{n}^{2}$ are non-trivial for all $n \in \mathbb{N}^{*}$, the energy of the dissipative system tends to 0 as $t \rightarrow \infty$. However, in general, the weights tend to 0 as $n \rightarrow \infty$, the observed quantity is weaker than the $V \times L^{2}(\mathcal{R})$-norm and the decay rate is not exponential.

It is important to underline that (1.9) holds under the same assumptions on the network needed for (1.8) to hold for the Dirichlet problem (1.7). Thus, no further analysis is required to establish the existence of the weights $\left\{c_{n}^{2}\right\}_{n \geq 1}$ in terms of the properties of the network. 
To derive decay properties out of (1.9), we view this inequality as a weak observability estimate in which the observed energy $E_{-}(0)$ is equal, roughly speaking, to $E_{*}^{D}(w, 0)+u_{1}^{(0)}(0)^{2}$. In practice we often take, if necessary, the lower convex envelop of $c_{n}^{2}$ instead of the weights $c_{n}^{2}$ in the definition of $E_{*}^{D}$ (see Section 3.1). The observed energy $E_{-}$is weaker than the $V \times L^{2}(\mathcal{R})$-norm of the initial data that would be required to prove exponential decay and, consequently, we obtain weaker decay rates. To obtain explicit decay rates out of this weak observability inequality we use an interpolation inequality which is a variant of the one from Bégout and Soria [7] and which is a generalization of Hölder's inequality. For this to be done we need to assume more regularity of the initial data. To be more precise we shall consider initial data $\left(u^{(0)}, u^{(1)}\right) \in X_{s}:=\left[D(\mathcal{A}), D\left(\mathcal{A}^{0}\right)\right]_{1-s}$ for $0<s<1 / 2$. In this way we deduce an interpolation inequality of the form

$$
1 \leq \Phi_{s}\left(\frac{E_{-}(0)}{C E_{u}(0)}\right) \frac{\left\|\left(u^{(0)}, u^{(1)}\right)\right\|_{X_{s}}^{2}}{C^{\prime} E_{u}(0)},
$$

where $\Phi_{s}$ is an increasing function which depends on $s$ and on the energy $E_{-}$under consideration.

The previous interpolation inequality implies

$$
E_{-}(0) \geq C E_{u}(0) \Phi_{s}^{-1}\left(\frac{E_{u}(0)}{C^{\prime}\left\|\left(u^{(0)}, u^{(1)}\right)\right\|_{X_{s}}^{2}}\right) .
$$

With (1.4) and (1.9), we obtain

$$
E_{u}(0)-E_{u}(T) \geq C E_{u}(0) \Phi_{s}^{-1}\left(\frac{E_{u}(0)}{C^{\prime}\left\|\left(u^{(0)}, u^{(1)}\right)\right\|_{X_{s}}^{2}}\right),
$$

which implies, by the semigroup property (see Ammari and Tucsnak [6])

$$
\forall t>0, E_{u}(t) \leq C \Phi_{s}\left(\frac{1}{t+1}\right)\left\|\left(u^{(0)}, u^{(1)}\right)\right\|_{X_{s}}^{2} .
$$

Obviously, the decay rate in (1.10) depends on the behaviour of the function $\Phi_{s}$ near 0 . Thus, in order to determine the explicit decay rate we need to have a sharp description of the function $\Phi_{s}$, which depends on $s$ and on the energies $E$ and $E_{-}$and thus, on the weights $\left(c_{n}^{2}\right)_{n}$ of (1.9) in an essential way. These weights depend on the topology of the network and the number theoretical properties of the lengths of the strings entering in it.

This approach allows getting in a systematic way decay rates for the energy of smooth solutions of the damped system as a consequence of the observability properties of the undamped one.

The analysis in this paper is limited to networks of strings with damping in one single end but the same methods, combined with the tools developped in [14], can be applied in other situations as, for instance:

a) networks of strings with damping in several end points;

b) networks with damping on end points and internal nodes;

c) networks of beams.

The paper is organized as follows. In the second section, we show how to pass from the observability inequality for the conservative problem (1.7) with Dirichlet boundary conditions at all exterior nodes to the weighted observability inequality (1.9) for (1.1). In section 3 we give an interpolation inequality which is a variant of the one by Bégout and Soria [7] and we apply it to obtain the explicit decay estimate of the energy. Finally we end up discussing some illustrative examples in section 4 . 
2. The weighted observability inequality. In this section, we prove that we can obtain a weighted observability estimate for $u$ solution of (1.1) directly from (1.8). First of all, we recall some results about system (1.7) with Dirichlet boundary conditions at all exterior nodes.

2.1. Preliminaries about the Dirichlet problem. Recall that if we suppose that $\left(\psi^{(0)}, \psi^{(1)}\right) \in$ $\tilde{H}$, then problem (1.7) admits a unique solution

$$
\psi \in C(0, T ; \tilde{V}) \cap C^{1}\left(0, T ; L^{2}(\mathcal{R})\right) .
$$

Denote by $\left(\tilde{\lambda}_{n}^{2}\right)_{n \geq 1}$ the sequence of eigenvalues corresponding to problem (1.7) and let $\left(\varphi_{n}^{D}\right)_{n \geq 1}$ be the corresponding eigenvectors forming an orthonormal basis of $L^{2}(\mathcal{R})$. We assume now that $\left(\psi^{(0)}, \psi^{(1)}\right) \in \tilde{H}$ and we set

$$
\psi^{(0)}=\sum_{n \geq 1} \psi_{0, n} \varphi_{n}^{D} \text { and } \psi^{(1)}=\sum_{n \geq 1} \psi_{1, n} \varphi_{n}^{D}
$$

where $\left(\tilde{\lambda}_{n} \psi_{0, n}\right)_{n},\left(\psi_{1, n}\right)_{n} \in l^{2}\left(\mathbb{N}^{*}\right)$.

In [14], a weighted observability inequality is derived, motivated by control problems. More precisely, it is shown that, under some conditions on the topology of the network and the lengths of the strings entering in it, for all $T>T_{0}$ (where $T_{0}>0$ is large enough), there exists a sequence of positive weights $\left\{c_{n}^{2}\right\}_{n \geq 1}$ and a positive constant $C$ such that for all solution $\psi$ of (1.7) it holds

$$
\sum_{n \geq 1} c_{n}^{2}\left(\tilde{\lambda}_{n}^{2} \psi_{0, n}^{2}+\psi_{1, n}^{2}\right) \leq C \int_{0}^{T}\left|\frac{\partial \psi_{1}}{\partial x}(0, t)\right|^{2} d t .
$$

Notice that, for this to be true, it is essential that the time $T_{0}>0$ to be large enough. More precisely, in [14] inequality (2.1) is proved in the case of general networks for $T>2 L$, where $L$ is the total length of the network. In the case of tree-like networks, $(2.1)$ is obtained by the D'Alembert representation formula and the weights $c_{n}^{2}$ are strictly positive for every $n \in \mathbb{N}^{*}$ if the tree is non-degenerate. In the case of stars, this comes down to impose irrationality conditions on the ratio of each pair of lengths. In the case of trees, the condition of non-degeneracy of the network is a natural extension of the irrationality one for stars and is also generically true (see $[9,12,14]$ for more details). In the case of general networks, $(2.1)$ is established by means of the Beurling-Malliavin Theorem under the assumption that all eigenfunctions have a non-trivial Neumann trace at the observation node. This condition is generically satisfied.

We set

$$
E_{*}^{D}(\psi, 0):=\frac{1}{2} \sum_{n \geq 1} c_{n}^{2}\left(\tilde{\lambda}_{n}^{2} \psi_{0, n}^{2}+\psi_{1, n}^{2}\right)
$$

the weighted energy for $\psi$ at time 0 . Note that $\left(E_{*}^{D}\right)^{\frac{1}{2}}$ defines a norm on $\tilde{H}$ because the weights $c_{n}^{2}$, according to the results in [14], are assumed to be positive. In the sequel, we assume that the network under consideration is such that the solutions $\psi$ of (1.7) satisfy (2.1).

2.2. The weighted observability inequality. In this section, we prove that we can obtain a weighted observability estimate for $u$ solution of (1.1) directly from (2.1). Thus, we assume that the network is such that $(2.1)$ holds and $\left(u^{(0)}, u^{(1)}\right) \in H$. 
We introduce $w$ solution of (1.7) with initial data $\left(w^{(0)}, w^{(1)}\right)=\left(u^{(0)}-u_{1}^{(0)}(0) \varphi, u^{(1)}\right)$, where $\varphi$ is a given smooth function satisfying

$$
\begin{cases}\varphi_{1}(0)=1 & \\ \varphi_{j_{v}}(v)=0 & \forall v \in \mathcal{D} \\ \varphi_{j}(v)=\varphi_{l}(v) & \forall j, l \in \mathcal{E}_{v}, v \in \mathcal{V}_{i n t} \\ \sum_{j \in \mathcal{E}_{v}} \frac{\partial \varphi}{\partial n_{j}}(v)=0 & \forall v \in \mathcal{V}_{i n t} .\end{cases}
$$

In this manner, the initial data $\left(w^{(0)}, w^{(1)}\right)$ belong to $\tilde{H}$, i. e. $w^{(0)}$ satisfies the Dirichlet boundary condition at the end 0 of $e_{1}$. Therefore, by hypothesis, $w$ satisfies (2.1).

We also consider $\epsilon$, the solution of the following non-homogeneous Dirichlet problem:

$$
\begin{cases}\frac{\partial^{2} \epsilon_{j}}{\partial t^{2}}(x, t)-\frac{\partial^{2} \epsilon_{j}}{\partial x^{2}}(x, t)=0 & \forall x \in\left(0, l_{j}\right), t>0, \forall j \in\{1, \ldots, N\}, \\ \epsilon_{j}(v, t)=\epsilon_{l}(v, t) & \forall j, l \in \mathcal{E}_{v}, v \in \mathcal{V}_{i n t}, t>0, \\ \sum_{j \in \mathcal{E}_{v}} \frac{\partial \epsilon_{j}}{\partial n_{j}}(v, t)=0 & \forall v \in \mathcal{V}_{i n t}, t>0, \\ \epsilon_{j_{v}}(v, t)=0 & \forall v \in \mathcal{D}, t>0, \\ \epsilon_{1}(0, t)=u_{1}(0, t) & t>0, \\ \epsilon(t=0)=u_{1}^{(0)}(0) \varphi, \frac{\partial \epsilon}{\partial t}(t=0)=0 . & \end{cases}
$$

Note that $\epsilon$ satisfies a non-homogeneous Dirichlet boundary condition at $x=0$. Actually it coincides with the value of the solution $u_{1}$ of (1.1) at that point. By Remark 1.1, we notice that $\partial u_{1} / \partial t(0,.) \in L^{2}(0, T)$, so that the non-homogeneous Dirichlet boundary condition belongs to $H^{1}(0, T)$.

In this way we have the decomposition

$$
u=w+\epsilon .
$$

In this section, we prove the following theorem

THEOREM 2.1. Assume that the network is such that the weighted observability inequality (2.1) is satisfied for the conservative system (1.7) with Dirichlet boundary conditions at all exterior nodes. We split up u, solution of (1.1), as (2.5) where $w$ is solution of (1.7) with initial data $\left(u^{(0)}-u_{1}^{(0)}(0) \varphi, u^{(1)}\right)$ and $\epsilon$ is solution of (2.4). We define $E_{*}(u, 0)$ by

$$
E_{*}(u, 0):=E_{*}^{D}(w, 0)+u_{1}^{(0)}(0)^{2},
$$

where $E_{*}^{D}(w, 0)$ is defined by (2.2). Then for all $T>T_{0}$, there exists $C_{T}>0$ such that all solution $u$ of (1.1) satisfies the weighted observability inequality

$$
E_{*}(u, 0) \leq C_{T} \int_{0}^{T}\left(\frac{\partial u_{1}}{\partial t}(0, t)\right)^{2} d t
$$

provided $\left(u^{(0)}, u^{(1)}\right) \in H$.

REMARK 2.2. Note that, according to Theorem 2.1, we transform the observability inequality for the Dirichlet problem (1.7) into a similar one for the dissipative one (1.1). Thus, in particular, estimate (2.7) holds under the same assumptions on the network that are needed for the Dirichlet problem and that are discussed in [14]. Some examples will be discussed in Section 4.

Note also that $\left(E_{*}(u, 0)\right)^{\frac{1}{2}}$ defines a norm in the space of initial data $\left(u^{(0)}, u^{(1)}\right) \in H$. Indeed, when $E_{*}(u, 0)$ vanishes, $u_{1}^{(0)}(0)=0$. Thus $\left(u^{(0)}, u^{(1)}\right) \in \tilde{H}$ and then $E_{*}(u, 0)=E_{*}^{D}(u, 0)$, and, by assumption, $\left(E_{*}^{D}(u, 0)\right)^{\frac{1}{2}}$ defines a norm in $\tilde{H}$. 
2.3. Proof of Theorem 2.1. Let $\left(u^{(0)}, u^{(1)}\right) \in H$. We decompose $u$ as in (2.5) where $w$ and $\epsilon$ solve (1.7) and (2.4) respectively. Therefore, $w$ is solution of (1.7) with initial data $\left(w^{(0)}, w^{(1)}\right)=\left(u^{(0)}-u_{1}^{(0)}(0) \varphi, u^{(1)}\right)$, and thus, by hypothesis, it satisfies $(2.1)$.

First, we have the following lemma

Lemma 2.3. For all $T>0$ there exists $C_{T}>0$ such that the solutions $u$ of (1.1) and $\epsilon$ of (2.4) satisfy the following estimate

$$
\int_{0}^{T}\left(\frac{\partial \epsilon_{1}}{\partial x}(0, t)\right)^{2} d t \leq C_{T}\left(\int_{0}^{T}\left(\frac{\partial u_{1}}{\partial t}(0, t)\right)^{2} d t+\left(u_{1}^{(0)}(0)\right)^{2}\right) .
$$

REMARK 2.4. Note that (2.8) holds for all networks since, in fact, it holds locally near the boundary of a single string. In this context we apply it along the string containing the extreme $x=0$, getting (2.8).

Proof. First of all, we easily show that the energy $E_{\epsilon}$ of the solution $\epsilon$ (defined as in (1.3)) satisfies

$$
\frac{d}{d t} E_{\epsilon}(t)=-\frac{\partial \epsilon_{1}}{\partial x}(0, t) \frac{\partial u_{1}}{\partial t}(0, t)
$$

and then for all $t>0$

$$
E_{\epsilon}(t)=E_{\epsilon}(0)-\int_{0}^{t} \frac{\partial \epsilon_{1}}{\partial x}(0, s) \frac{\partial u_{1}}{\partial t}(0, s) d s .
$$

Therefore, by using Cauchy's inequality, we obtain

$$
E_{\epsilon}(t) \leq E_{\epsilon}(0)+\frac{1}{4} \int_{0}^{t}\left(\frac{\partial \epsilon_{1}}{\partial x}(0, s)\right)^{2} d s+\int_{0}^{t}\left(\frac{\partial u_{1}}{\partial t}(0, s)\right)^{2} d s .
$$

Secondly, for all $t \in(0, T]$, multiplying the wave equation satisfied by $\epsilon_{1}$ by $\left(l_{1}-x\right) \frac{\partial \epsilon_{1}}{\partial x}$ and by integrating on $\left(0, l_{1}\right) \times(0, t)$, we have

$$
\int_{0}^{t} \int_{0}^{l_{1}}\left(l_{1}-x\right) \frac{\partial \epsilon_{1}}{\partial x} \frac{\partial^{2} \epsilon_{1}}{\partial t^{2}} d x d s-\int_{0}^{t} \int_{0}^{l_{1}}\left(l_{1}-x\right) \frac{\partial \epsilon_{1}}{\partial x} \frac{\partial^{2} \epsilon_{1}}{\partial x^{2}} d x d s=0 .
$$

By integration by parts, we have

$$
\begin{aligned}
\int_{0}^{t} \int_{0}^{l_{1}}\left(l_{1}-x\right) \frac{\partial \epsilon_{1}}{\partial x} \frac{\partial^{2} \epsilon_{1}}{\partial t^{2}} d x d s= & -\int_{0}^{t} \int_{0}^{l_{1}}\left(l_{1}-x\right) \frac{\partial^{2} \epsilon_{1}}{\partial x t} \frac{\partial \epsilon_{1}}{\partial t} d x d s \\
& +\left.\int_{0}^{l_{1}}\left(l_{1}-x\right) \frac{\partial \epsilon_{1}}{\partial x}(s, x) \frac{\partial \epsilon_{1}}{\partial t}(s, x) d x\right|_{0} ^{t} \\
= & -\frac{1}{2} \int_{0}^{t} \int_{0}^{l_{1}}\left(\frac{\partial \epsilon_{1}}{\partial t}\right)^{2} d x d s+\frac{l_{1}}{2} \int_{0}^{t}\left(\frac{\partial u_{1}}{\partial t}(0, s)\right)^{2} d s \\
& +\int_{0}^{l_{1}}\left(l_{1}-x\right) \frac{\partial \epsilon_{1}}{\partial x}(t, x) \frac{\partial \epsilon_{1}}{\partial t}(t, x) d x
\end{aligned}
$$

because $\frac{\partial \epsilon}{\partial t}(t=0)=0$, and

$$
\begin{aligned}
\int_{0}^{t} \int_{0}^{l_{1}}\left(l_{1}-x\right) \frac{\partial \epsilon_{1}}{\partial x} \frac{\partial^{2} \epsilon_{1}}{\partial x^{2}} d x d s & =\frac{1}{2} \int_{0}^{t} \int_{0}^{l_{1}}\left(l_{1}-x\right) \frac{\partial}{\partial x}\left(\left(\frac{\partial \epsilon_{1}}{\partial x}\right)^{2}\right) d x d s \\
& =\frac{1}{2} \int_{0}^{t} \int_{0}^{l_{1}}\left(\frac{\partial \epsilon_{1}}{\partial x}\right)^{2} d x d s-\frac{l_{1}}{2} \int_{0}^{t}\left(\frac{\partial \epsilon_{1}}{\partial x}(0, s)\right)^{2} d s
\end{aligned}
$$


Therefore, we obtain

$$
\begin{gathered}
-\frac{1}{2} \int_{0}^{t} \int_{0}^{l_{1}}\left(\frac{\partial \epsilon_{1}}{\partial t}\right)^{2} d x d s+\frac{l_{1}}{2} \int_{0}^{t}\left(\frac{\partial u_{1}}{\partial t}(0, s)\right)^{2} d s+\int_{0}^{l_{1}}\left(l_{1}-x\right) \frac{\partial \epsilon_{1}}{\partial x}(t, x) \frac{\partial \epsilon_{1}}{\partial t}(t, x) d x \\
-\frac{1}{2} \int_{0}^{t} \int_{0}^{l_{1}}\left(\frac{\partial \epsilon_{1}}{\partial x}\right)^{2} d x d s+\frac{l_{1}}{2} \int_{0}^{t}\left(\frac{\partial \epsilon_{1}}{\partial x}(0, s)\right)^{2} d s=0
\end{gathered}
$$

and thus

$$
\int_{0}^{t}\left(\frac{\partial \epsilon_{1}}{\partial x}(0, s)\right)^{2} d s \leq \frac{2}{l_{1}} \int_{0}^{t} E_{\epsilon}(s) d s+2 E_{\epsilon}(t) .
$$

By grouping (2.9) and (2.10), we find

$$
E_{\epsilon}(t) \leq E_{\epsilon}(0)+\frac{1}{2 l_{1}} \int_{0}^{t} E_{\epsilon}(s) d s+\frac{1}{2} E_{\epsilon}(t)+\int_{0}^{t}\left(\frac{\partial u_{1}}{\partial t}(0, s)\right)^{2} d s .
$$

That is to say

$$
E_{\epsilon}(t) \leq 2 E_{\epsilon}(0)+\frac{1}{l_{1}} \int_{0}^{t} E_{\epsilon}(s) d s+2 \int_{0}^{t}\left(\frac{\partial u_{1}}{\partial t}(0, s)\right)^{2} d s
$$

By Gronwall's lemma, we obtain that, for all $t \in(0, T]$,

$$
E_{\epsilon}(t) \leq C_{T}\left(E_{\epsilon}(0)+\int_{0}^{T}\left(\frac{\partial u_{1}}{\partial t}(0, s)\right)^{2} d s\right)
$$

where $C_{T}$ depends on $T$. By inserting this expression into (2.10), we find

$$
\int_{0}^{T}\left(\frac{\partial \epsilon_{1}}{\partial x}(0, t)\right)^{2} d t \leq C_{T}\left(E_{\epsilon}(0)+\int_{0}^{T}\left(\frac{\partial u_{1}}{\partial t}(0, t)\right)^{2} d t\right) .
$$

But

$$
E_{\epsilon}(0)=\left(u_{1}^{(0)}(0)\right)^{2}\left(\frac{1}{2} \sum_{j=1}^{N} \int_{0}^{l_{j}}\left(\frac{\partial \varphi_{j}}{\partial x}(x)\right)^{2} d x\right)=C\left(u_{1}^{(0)}(0)\right)^{2},
$$

where $C$ is a constant, since $\varphi$ is given as in (2.3), independently of the solution under consideration. This proves (2.8).

Let us now return to the proof of Theorem 2.1.

Since $w$ satisfies (2.1), we have

$$
E_{*}^{D}(w, 0)=E_{*}^{D}(w, t) \leq C \int_{0}^{T}\left(\frac{\partial w_{1}}{\partial x}(0, t)\right)^{2} d t
$$

where $E_{*}^{D}(w, 0)$ is defined as in $(2.2)$ for $w$ solution of $(1.7)$ with initial data $\left(u^{(0)}-u_{1}^{(0)}(0) \varphi, u^{(1)}\right)$.

Moreover, we have

$$
\int_{0}^{T}\left(\frac{\partial w_{1}}{\partial x}(0, t)\right)^{2} d t \leq C \int_{0}^{T}\left(\left(\frac{\partial u_{1}}{\partial x}(0, t)\right)^{2}+\left(\frac{\partial \epsilon_{1}}{\partial x}(0, t)\right)^{2}\right) d t .
$$


Therefore, with (2.8) and (2.11), we obtain

$$
E_{*}^{D}(w, 0)=E_{*}^{D}(w, t) \leq C_{T} \int_{0}^{T}\left(\left(\frac{\partial u_{1}}{\partial t}(0, t)\right)^{2}+\left(\frac{\partial u_{1}}{\partial x}(0, t)\right)^{2}\right) d t+C_{T}\left(u_{1}^{(0)}(0)\right)^{2} .
$$

We recall that $E_{*}(u, 0)$ is given by $(2.6)$ where $E_{*}^{D}(w, 0)$ is defined by $(2.2)$. Then, $(2.12)$ becomes

$$
E_{*}(u, 0) \leq C_{T} \int_{0}^{T}\left(\left(\frac{\partial u_{1}}{\partial t}(0, t)\right)^{2}+\left(\frac{\partial u_{1}}{\partial x}(0, t)\right)^{2}\right) d t+C_{T}\left(u_{1}^{(0)}(0)\right)^{2} .
$$

In fact, we can remove the last term in the right hand side of (2.13).

LEMma 2.5. For T large enough, the solutions $u$ of (1.1) satisfy

$$
E_{*}(u, 0) \leq C_{T} \int_{0}^{T}\left(\left(\frac{\partial u_{1}}{\partial t}(0, t)\right)^{2}+\left(\frac{\partial u_{1}}{\partial x}(0, t)\right)^{2}\right) d t
$$

for a positive constant $C_{T}$ depending on $T$.

Proof. In view of (2.13) it is sufficient to show that there exists a positive constant $C>0$ such that

$$
\left|u_{1}^{(0)}(0)\right|^{2} \leq C \int_{0}^{T}\left(\left(\frac{\partial u_{1}}{\partial t}(0, t)\right)^{2}+\left(\frac{\partial u_{1}}{\partial x}(0, t)\right)^{2}\right) d t .
$$

We argue by contradiction. If this is not the case, there exists a sequence of solutions $u_{n}$ such that

$$
\left|u_{n, 1}^{(0)}(0)\right|=1, \forall n \in \mathbb{N}
$$

and

$$
\int_{0}^{T}\left(\left(\frac{\partial u_{n, 1}}{\partial t}(0, t)\right)^{2}+\left(\frac{\partial u_{n, 1}}{\partial x}(0, t)\right)^{2}\right) d x \rightarrow 0, \text { as } n \rightarrow+\infty .
$$

In view of this and (2.13), we deduce that $E_{*}\left(u_{n}, 0\right)$ are uniformly bounded. Passing weakly to the limit in the Hilbert space $\mathcal{H}$, defined as the closure of $V$ with respect to the norm $\left(E_{*}\left(u_{n}, 0\right)\right)^{\frac{1}{2}}$, we obtain a limit solution $u$ such that

$$
\left|u_{1}^{(0)}(0)\right|=1
$$

and

$$
\int_{0}^{T}\left(\left(\frac{\partial u_{1}}{\partial t}(0, t)\right)^{2}+\left(\frac{\partial u_{1}}{\partial x}(0, t)\right)^{2}\right) d x=0 .
$$

The fact that (2.16) holds is a consequence of the compactness of the trace operator from $\mathcal{R}$ to $\mathbb{R}$ (it is in fact an operator of rank one). On the other hand, (2.17) holds as a consequence of the weak lower semicontinuity. But, by unique continuation, it is easy to see, in view of (2.17), that for all $T>2 L$, the whole limit solution $u$ vanishes. Indeed, we obtain by $(2.17)$

$$
\frac{\partial u_{1}}{\partial t}(0, t)=\frac{\partial u_{1}}{\partial x}(0, t)=0,
$$


and therefore $\partial u / \partial t$ solves system (1.7) with initial data $\left(u^{(1)}, \partial^{2} u^{(0)} / \partial x^{2}\right)$. Then, we can apply (2.1) to obtain

$$
u^{(1)}=\frac{\partial^{2} u^{(0)}}{\partial x^{2}}=0 .
$$

Consequently, $\partial u / \partial t=0$ on $\mathcal{R} \times(0, T)$, and thus $u$ is independent of $t$. Thus $u=0$ on $\mathcal{R} \times(0, T)$ since it is harmonic on the network and fulfills the Dirichlet boundary condition. This contradicts (2.16).

This lemma proves Theorem 2.1, because $u$ is solution of (1.1).

\section{The stabilization result.}

3.1. An interpolation inequality. In this subsection, we give an interpolation result similar to the one of [7].

Let $m \in[0,1), 0<s<1 / 2$ and assume that

$$
\begin{gathered}
\omega:(m, \infty) \rightarrow(0, \omega(m)) \text { is a convex and decreasing function with } \omega(\infty)=0, \\
\Phi_{s}:(0, \omega(m)) \rightarrow(0, \infty) \text { is a concave and increasing function with } \Phi_{s}(0)=0, \\
\forall t \in[1, \infty), 1 \leq \Phi_{s}(\omega(t)) t^{2 s}
\end{gathered}
$$

The function $t \mapsto \frac{1}{t} \Phi_{s}^{-1}(t)$ is nondecreasing on $(0,1)$.

Before stating the needed interpolation inequality, we recall the inverse Jensen's inequality, which is the inverse version of the classical Jensen's inequality (see Lemma 2.4 from [7] and Rudin $[22])$.

Lemma 3.1 (Inverse Jensen's inequality). Let $(\Omega, \Upsilon, \nu)$ be a measure space such that $\nu(\Omega)=1$ and let $-\infty \leq a<b \leq \infty$. Assume that

1) $\varphi:(a, b) \rightarrow \mathbb{R}$ is a concave function,

2) $g \in L^{1}(\Omega, \Upsilon, \nu)$ is such that for almost every $x \in \Omega, g(x) \in(a, b)$.

Then $\varphi(g)_{+} \in L^{1}(\Omega, \Upsilon, \nu)$ and

$$
\int_{\Omega} \varphi(g) d \nu \leq \varphi\left(\int_{\Omega} g d \nu\right)
$$

Under the conditions (3.2)-(3.3), we have the following result which is a generalized Hölder's inequality, a variant of Theorem 2.1 given in [7]:

THEOREM 3.2. Let $\left(\omega, \Phi_{s}\right)$ be as above satisfying (3.1)-(3.3). Then for any $f=\left(f_{n}\right)_{n \in \mathbb{N}^{*}} \in$ $l^{1}\left(\mathbb{N}^{*}\right), f \neq 0$, we have

$$
1 \leq \Phi_{s}\left(\frac{\sum_{n \geq 1}\left|f_{n}\right| \omega(n)}{\sum_{n \geq 1}\left|f_{n}\right|}\right) \frac{\sum_{n \geq 1}\left|f_{n}\right| n^{2 s}}{\sum_{n \geq 1}\left|f_{n}\right|}
$$


as soon as $\left(f_{n} \omega(n)\right)_{n} \in l^{1}\left(\mathbb{N}^{*}\right)$ and $\left(f_{n} n^{2 s}\right)_{n} \in l^{1}\left(\mathbb{N}^{*}\right)$.

Proof. The proof is similar to that of Theorem 2.1 of [7]. We give it for the sake of completeness. By (3.3) and Cauchy-Schwarz's inequality, we have

$$
\begin{aligned}
1=\left(\sum_{n \geq 1} \frac{\left|f_{n}\right|}{\sum_{n \geq 1}\left|f_{n}\right|}\right)^{2} & \leq\left(\sum_{n \geq 1} \frac{\left|f_{n}\right|}{\sum_{n \geq 1}\left|f_{n}\right|} \Phi_{s}^{\frac{1}{2}}(\omega(n)) n^{s}\right)^{2} \\
& \leq\left(\sum_{n \geq 1} \frac{\left|f_{n}\right|}{\sum_{n \geq 1}\left|f_{n}\right|} \Phi_{s}(\omega(n))\right)\left(\sum_{n \geq 1} \frac{\left|f_{n}\right|}{\sum_{n \geq 1}\left|f_{n}\right|} n^{2 s}\right) .
\end{aligned}
$$

Now, we apply Lemma 3.1 with $\varphi=\Phi_{s}$ a concave function, $g=\omega$ and the discrete measure $\nu=\sum_{n \geq 1} \frac{\left|f_{n}\right|}{\sum_{n \geq 1}\left|f_{n}\right|} \delta_{n}$, by noticing that

$$
\left(\sum_{n \geq 1} \frac{\left|f_{n}\right|}{\sum_{n \geq 1}\left|f_{n}\right|}\right)=1 .
$$

We then obtain (3.5).

We now give some examples of pairs $\left(\omega, \Phi_{s}\right)$ satisfying (3.1)-(3.4):

EXAMPLE 3.3. 1. If

$$
\omega(t)=\frac{c}{t^{p}},
$$

for some $p \geq 1$, we can take $\Phi_{s}$ of the form

$$
\Phi_{s}(t)=\left(\frac{t}{c}\right)^{\frac{2 s}{p}}
$$

We can easily prove that $\left(\omega, \Phi_{s}\right)$ satisfy (3.1)-(3.2) with $m=0$ and (3.3)-(3.4).

2. If

$$
\omega(t)=C e^{-A t}
$$

where $A>2(2 s+1)$ and $C>0$, we can take $\Phi_{s}$ of the form

$$
\Phi_{s}(t)=\left(\frac{A}{\ln \left(\frac{C}{t}\right)}\right)^{2 s} .
$$

We can easily prove that $\Phi_{s}$ is an increasing function on $(0, \omega(0))$, a concave function on $(0, \omega(1 / 2))$ (because $A>2(2 s+1)$ ), that $t \mapsto \frac{1}{t} \Phi_{s}^{-1}(t)$ is nondecreasing on $(0,1)$ and that the pair $\left(\omega, \Phi_{s}\right)$ satisfies (3.3) on $[1, \infty)$. Thus $\left(\omega, \Phi_{s}\right)$ satisfy (3.1)-(3.4) with $m=1 / 2$.

In the application of the interpolation inequality of Theorem 3.2 to our stabilization problem the weight $\omega$ is determined by the weights $\left(c_{n}^{2}\right)_{n}$ in (2.2). However, notice that, in general, the weights $c_{n}^{2}$ may degenerate fast and, consequently, we have to work in a more general context. 
Moreover, in principle, $\left(c_{n}^{2}\right)_{n}$ does not necessarily satisfy the convexity or the monotony property in (3.1). To ensure that this assumption is satisfied, we introduce the notion of the lower convex envelop of a sequence $\left(u_{n}\right)_{n}$ satisfying $\liminf \operatorname{in}_{n \rightarrow \infty} u_{n}=0$. Roughly, it is the "nearest" convex and decreasing function $\omega(n)$ satisfying $0<\omega(n) \leq u_{n}$ for all $n \in \mathbb{N}$. The existence of this function is guaranteed by the following lemma:

Lemma 3.4. ([7]) Let $-\infty<a<b \leq \infty$ and let $\epsilon:[a, b) \rightarrow(0, \infty)$ be a continuous function such that $\liminf _{t \nearrow b} \epsilon(t)=0$. Then there exists a convex function $\varphi \in C_{b}^{1}([a, b) ; \mathbb{R})$ such that $0<\varphi \leq \epsilon$ and $\varphi^{\prime}<0$ on $[a, b)$.

The proof of this lemma can be found in [7].

Now, we show how to construct the weight $\omega$ satisfying (3.1) from $\left(u_{n}\right)_{n \in \mathbb{N}} \subset(0, \infty)$ such that $\liminf _{n \rightarrow \infty} u_{n}=0$. Let $\epsilon \in C([0, \infty) ; \mathbb{R})$ be such that $0<\epsilon(n) \leq u_{n}$, for any $n \in \mathbb{N}$. Let $\varphi \in C([0, \infty) ; \mathbb{R})$ be a decreasing and convex function such that for any $t \geq 0,0<\varphi(t) \leq \epsilon(t)$ (which exists by the previous lemma) and consider $\mathcal{C} \subset[1, \infty) \times[0, \infty)$ the closure of the convex envelop of the set $\left\{\left(n, u_{n}\right) ; n \in \mathbb{N}\right\}$. Finally, fix arbitrarily $t \geq 1$. Then the set $\mathcal{C}_{t}=\mathcal{C} \cap(\{t\} \times \mathbb{R})$ is nonempty, closed and the previous lemma ensures that for any $s_{t} \in \mathbb{R}$ such that $\left(t, s_{t}\right) \in \mathcal{C}_{t}$,

$$
0<\varphi(t) \leq s_{t}
$$

So by compactness, we may define the function $\omega$ as

$$
\forall t \geq 1, \omega(t)=\min \left\{s_{t} ;\left(t, s_{t}\right) \in \mathcal{C}_{t}\right\}
$$

Finally we extend $\omega$ as a decreasing, continuous and convex function on $[0,1]$. Therefore, $\omega$ satisfies (3.1) with $m=0$. This function $\omega$ is called the lower convex envelop of the sequence $\left(u_{n}\right)_{n}$.

In the sequel, assuming that the weights in (2.1) are such that $\liminf _{n \rightarrow \infty} c_{n}=0$ and $c_{n} \neq 0$ for all $n \in \mathbb{N}^{*}$, we choose the function $\omega$ as follows

$$
\omega \text { is the lower convex envelop of the sequence }\left(1,\left(c_{n}^{2}\right)_{n \geq 1}\right) .
$$

3.2. The main results. In this subsection, we assume that the network is such that the weighted observability inequality (2.1) holds, for every solution of problem (1.7). Therefore, by Theorem 2.1, $u$ satisfies (2.7). Let us make some remarks:

REMARK 3.5. 1. If for all $n \in \mathbb{N}^{*}$, we have $c_{n}^{2}>0$, then the energy $E_{u}$ tends to 0 as $t \rightarrow \infty$.

2. If there exists a positive constant $c$ such that for all $n \in \mathbb{N}^{*}, c_{n}^{2} \geq c>0$, then the energy of $u$ solution of (1.1) is exponentially decreasing. Unfortunately this does not hold in general except, for example, for the simplest network which consists simply of a single string and for trees with all but one damped ends ([20]), but never for non-trivial networks with one single damped end.

The statement in the first point can be proved by the La Salle's invariance principle and the second one by a classical energy method combining the observability inequality (2.7) and the energy dissipation law (1.4) (see for instance [20]).

Before stating the main result of this paper, let us give a technical lemma which will be used in the sequel. For that, we need to define $X_{s}$ the interpolation space between $D(\mathcal{A})$ and $D\left(\mathcal{A}^{0}\right)$ :

$$
X_{s}:=\left[D(\mathcal{A}), D\left(\mathcal{A}^{0}\right)\right]_{1-s},
$$

where $0<s<1 / 2$. Note that if $s=0$ then $X_{0}=D\left(\mathcal{A}^{0}\right)=V \times L^{2}(\mathcal{R})$ and if $s=1$ then $X_{1}=D(\mathcal{A})$. More precisely we can identify the space $X_{s}$ : 
Lemma 3.6. For $0<s<1 / 2$,

$$
X_{s}=\left(V \cap \prod_{j} H^{1+s}\left(0, l_{j}\right)\right) \times \prod_{j} H^{s}\left(0, l_{j}\right) .
$$

Proof. First $D(\mathcal{A})$ is a dense subset of $V \times L^{2}(\mathcal{R})$ and $\left(V \cap \prod_{j=1}^{N} H^{2}\left(0, l_{j}\right)\right) \times V$ is a dense subset of $V \times L^{2}(\mathcal{R})$ with $D(\mathcal{A}) \subset\left(V \cap \prod_{j=1}^{N} H^{2}\left(0, l_{j}\right)\right) \times V$. Then $\left[D(\mathcal{A}), V \times L^{2}(\mathcal{R})\right]_{1-s}$ is a subset of

$$
\left[\left(V \cap \prod_{j=1}^{N} H^{2}\left(0, l_{j}\right)\right) \times V, V \times L^{2}(\mathcal{R})\right]_{1-s}
$$

with the same norms.

But

$$
\left[\left(V \cap \prod_{j=1}^{N} H^{2}\left(0, l_{j}\right)\right) \times V, V \times L^{2}(\mathcal{R})\right]_{1-s}=\left(V \cap \prod_{j=1}^{N} H^{1+s}\left(0, l_{j}\right)\right) \times \prod_{j=1}^{N} H^{s}\left(0, l_{j}\right),
$$

and thus, if $D(\mathcal{A})$ is dense in $\left(V \cap \prod_{j=1}^{N} H^{1+s}\left(0, l_{j}\right)\right) \times \prod_{j=1}^{N} H^{s}\left(0, l_{j}\right)$, then, by a classical result of [23],

$$
X_{s}=\left[\left(V \cap \prod_{j=1}^{N} H^{2}\left(0, l_{j}\right)\right) \times V, V \times L^{2}(\mathcal{R})\right]_{1-s}=\left(V \cap \prod_{j=1}^{N} H^{1+s}\left(0, l_{j}\right)\right) \times \prod_{j=1}^{N} H^{s}\left(0, l_{j}\right) .
$$

To verify that $D(\mathcal{A})$ is dense in $\left(V \cap \prod_{j=1}^{N} H^{1+s}\left(0, l_{j}\right)\right) \times \prod_{j=1}^{N} H^{s}\left(0, l_{j}\right)$, we note that $D(\Delta) \times$ $\prod_{j=1}^{N} \mathcal{D}\left(0, l_{j}\right) \subset D(\mathcal{A})$, where

$$
D(\Delta):=\left\{u \in V \cap \prod_{j=1}^{N} H^{2}\left(0, l_{j}\right): \sum_{j \in \mathcal{E}_{v}} \frac{\partial u_{j}}{\partial n_{j}}(v)=0, \forall v \in \mathcal{V}_{i n t} ; \frac{\partial u_{1}}{\partial x}(0)=0\right\}
$$

and $\mathcal{D}\left(0, l_{j}\right)$ is the space of $C^{\infty}$ functions with compact support in $\left(0, l_{j}\right)$. As $\prod_{j=1}^{N} \mathcal{D}\left(0, l_{j}\right)$ is dense in $\prod_{j=1}^{N} H^{s}\left(0, l_{j}\right)$ (because $\left.0<s<1 / 2\right)$, it remains to show that $D(\Delta)$ is dense in $V \cap \prod_{j=1}^{N} H^{1+s}\left(0, l_{j}\right)$. For that, let $\xi \in V \cap \prod_{j=1}^{N} H^{1+s}\left(0, l_{j}\right)$. For $j \in\{1, \ldots, N\}$, we take

$$
\hat{\xi}_{j}=\xi_{j}-\xi_{j}(0) \eta_{j}-\xi_{j}\left(l_{j}\right) \tilde{\eta}_{j},
$$

where $\eta_{j}, \tilde{\eta}_{j}$ are $C^{\infty}$ functions satisfying

$$
\eta_{j}(0)=1 \text { near } 0, \quad \eta_{j}\left(l_{j}\right)=0 \text { near } l_{j} \quad \text { and } \quad \tilde{\eta}_{j}(0)=0 \text { near } 0, \quad \tilde{\eta}_{j}\left(l_{j}\right)=1 \text { near } l_{j} .
$$

Then $\hat{\xi}_{j} \in H_{0}^{1+s}\left(0, l_{j}\right)$. As $\mathcal{D}\left(0, l_{j}\right)$ is dense into $H_{0}^{1+s}\left(0, l_{j}\right)$, there exits a sequence $\hat{\xi}_{n, j} \in \mathcal{D}\left(0, l_{j}\right)$ such that $\hat{\xi}_{n, j} \rightarrow \hat{\xi}_{j}$ in $H_{0}^{1+s}\left(0, l_{j}\right)$ when $n \rightarrow \infty$. Setting

$$
\xi_{n, j}:=\hat{\xi}_{n, j}+\xi_{j}(0) \eta_{j}+\xi_{j}\left(l_{j}\right) \tilde{\eta}_{j}
$$


we see that $\xi_{n}=\left(\xi_{n, j}\right)_{j=1, \ldots, N} \in D(\Delta)$ and $\xi_{n} \rightarrow \xi$ in $\prod_{j=1}^{N} H^{1+s}\left(0, l_{j}\right)$.

As $D(\Delta)$ is dense in $V \cap \prod_{j=1}^{N} H^{1+s}\left(0, l_{j}\right), D(\mathcal{A})$ is dense in $\left(V \cap \prod_{j=1}^{N} H^{1+s}\left(0, l_{j}\right)\right) \times \prod_{j=1}^{N} H^{s}\left(0, l_{j}\right)$, which finishes the proof. $\square$

Then we have the following lemma:

LemmA 3.7. Assume that $\left(u^{(0)}, u^{(1)}\right)$ belongs to $X_{s}$, where $0<s<1 / 2$, and $\left(w^{(0)}, w^{(1)}\right)=$ $\left(u^{(0)}-u_{1}^{(0)}(0) \varphi, u^{(1)}\right)$ where $\varphi$ is a given smooth function satisfying (2.3). Then there exists a positive constant $C$ such that

$$
\left\|\left(w^{(0)}, w^{(1)}\right)\right\|_{D\left(\mathcal{A}_{D}^{s}\right)}^{2}+\left|u_{1}^{(0)}(0)\right|^{2} \leq C\left\|\left(u^{(0)}, u^{(1)}\right)\right\|_{X_{s}}^{2},
$$

where $D\left(\mathcal{A}_{D}^{s}\right)$ is the domain of the operator $\mathcal{A}^{s}$ with Dirichlet boundary conditions at all exterior nodes.

Proof. First, we know that there exists $C>0$ such that

$$
\left\|\left(w^{(0)}, w^{(1)}\right)\right\|_{D\left(\mathcal{A}_{D}^{s}\right)}^{2} \leq C\left\|\left(w^{(0)}, w^{(1)}\right)\right\|_{\left(\tilde{V} \cap \prod_{j=1}^{N} H^{1+s}\left(0, l_{j}\right)\right) \times \prod_{j=1}^{N} H^{s}\left(0, l_{j}\right)}^{2}
$$

by interpolation (see [23] for example).

This estimate leads to the existence of a positive constant $C$ such that

$$
\left\|\left(w^{(0)}, w^{(1)}\right)\right\|_{D\left(\mathcal{A}_{D}^{s}\right)}^{2} \leq C\left\|\left(w^{(0)}, w^{(1)}\right)\right\|_{\left(V \cap \prod_{j=1}^{N} H^{1+s}\left(0, l_{j}\right)\right) \times \prod_{j=1}^{N} H^{s}\left(0, l_{j}\right)}^{2},
$$

because $w^{(0)} \in V \cap \tilde{V}$.

The Sobolev's injection theorem and the fact that $\left(w^{(0)}, w^{(1)}\right)=\left(u^{(0)}-u_{1}^{(0)}(0) \varphi, u^{(1)}\right)$ imply that there exists $C>0$ such that

$$
\left\|w^{(0)}\right\|_{V \cap \prod_{j=1}^{N} H^{1+s}\left(0, l_{j}\right)}^{2}+\left|u_{1}^{(0)}(0)\right|^{2} \leq C\left\|u^{(0)}\right\|_{\prod_{j=1}^{N} H^{1+s}\left(0, l_{j}\right)}^{2} .
$$

By definition of $w^{(1)}$, we have also

$$
\left\|w^{(1)}\right\|_{\prod_{j=1}^{N} H^{s}\left(0, l_{j}\right)}^{2}=\left\|u^{(1)}\right\|_{\prod_{j=1}^{N} H^{s}\left(0, l_{j}\right)}^{2} .
$$

Moreover, by the continuous injection of

$$
\left[D(\mathcal{A}), D\left(\mathcal{A}^{0}\right)\right]_{1-s}=X_{s}
$$

in

$$
\left[\prod_{j=1}^{N} H^{2}\left(0, l_{j}\right) \times \prod_{j=1}^{N} H^{1}\left(0, l_{j}\right), \prod_{j=1}^{N} H^{1}\left(0, l_{j}\right) \times L^{2}(\mathcal{R})\right]_{1-s}=\prod_{j=1}^{N} H^{1+s}\left(0, l_{j}\right) \times \prod_{j=1}^{N} H^{s}\left(0, l_{j}\right),
$$

there exists $C>0$ such that

$$
\left\|\left(u^{(0)}, u^{(1)}\right)\right\|_{\prod_{j=1}^{N} H^{1+s}\left(0, l_{j}\right) \times \prod_{j=1}^{N} H^{s}\left(0, l_{j}\right)}^{2} \leq C\left\|\left(u^{(0)}, u^{(1)}\right)\right\|_{X_{s}}^{2} .
$$


The estimates (3.7)-(3.10) prove this lemma. $\square$

The main results of the paper are the following

THEOREM 3.8. Assume that the weighted observability inequality (2.1) holds for every solution of (1.7) with $\liminf \operatorname{in}_{n \rightarrow \infty} c_{n}=0$ and $c_{n} \neq 0$ for all $n \in \mathbb{N}^{*}$. Let $\omega$ be defined by (3.6). Assume that the initial data $\left(u^{(0)}, u^{(1)}\right)$ belong to $X_{s}$, characterized by Lemma 3.6, where $0<s<1 / 2$. Let $\Phi_{s}$ be a function such that the pair $\left(\omega, \Phi_{s}\right)$ satisfies (3.1)-(3.4). Then there exists a constant $C>0$ such that the corresponding solution $u$ of (1.1) verifies

$$
\forall t \geq 0, E_{u}(t) \leq C \Phi_{s}\left(\frac{1}{t+1}\right)\left\|\left(u^{(0)}, u^{(1)}\right)\right\|_{X_{s}}^{2} .
$$

REMARK 3.9. We see that the decay rate of the energy directly depends on the behaviour of the interpolation function $\Phi_{s}$ near 0 and thus of $\omega$ and of the weights $c_{n}^{2}$ as $n \rightarrow \infty$.

Proof. We split up $u$ as in (2.5) and we decompose $w^{(0)}=u^{(0)}-u_{1}^{(0)}(0) \varphi$ and $w^{(1)}=u^{(1)}$ as

$$
w^{(0)}=u^{(0)}-u_{1}^{(0)}(0) \varphi=\sum_{n \geq 1} w_{0, n} \varphi_{n}^{D} \text { and } w^{(1)}=u^{(1)}=\sum_{n \geq 1} w_{1, n} \varphi_{n}^{D},
$$

where $\left(\tilde{\lambda}_{n} w_{0, n}\right)_{n},\left(w_{1, n}\right)_{n} \in l^{2}\left(\mathbb{N}^{*}\right)$. We write

$$
E_{-}(0)=\sum_{n \geq 1} u_{n} \omega(n)
$$

where $\left(u_{n}\right)_{n \in \mathbb{N}^{*}} \in l^{1}\left(\mathbb{N}^{*} ; \mathbb{R}\right)$ is defined by

$$
\forall n \geq 2, u_{n}=\frac{1}{2}\left(\tilde{\lambda}_{n-1}^{2} w_{0, n-1}^{2}+w_{1, n-1}^{2}\right)
$$

and

$$
u_{1}=u_{1}^{(0)}(0)^{2}
$$

Observe that, by the construction (3.6) of $\omega$, we have

$$
E_{-}(u, 0) \leq E_{*}(u, 0) .
$$

Then, by (1.4) and (2.7) of Theorem 2.1, we have

$$
E_{u}(0)-E_{u}(T)=\int_{0}^{T}\left(\frac{\partial u_{1}}{\partial t}(0, t)\right)^{2} d t \geq C E_{-}(0) .
$$

Assume further that $\left(u^{(0)}, u^{(1)}\right) \in X_{s}$. We define

$$
E_{+}(0)=\sum_{n \geq 1} u_{n} n^{2 s} .
$$

It follows from Theorem 3.2 (applied to the function $f=u$ and the weight $\omega$ ) that

$$
1 \leq \Phi_{s}\left(\frac{E_{-}(0)}{\sum_{n \geq 1} u_{n}}\right) \frac{E_{+}(0)}{\sum_{n \geq 1} u_{n}} .
$$


By the so-called Weyl's formula (see for instance Theorem 1.3 of [1], Proposition 6.2 of [14] or $[19,24])$, we have

$$
\tilde{\lambda}_{k} \sim \frac{k \pi}{L}
$$

and thus, there exist $c_{1}, c_{2}>0$ such that, for $n$ large enough, we have

$$
c_{1} \frac{(n+1) \pi}{L} \leq \tilde{\lambda}_{n} \leq c_{2} \frac{(n+1) \pi}{L} .
$$

Therefore, we have

$$
\begin{aligned}
E_{+}(0) & =\frac{1}{2}\left(\frac{L}{\pi}\right)^{2 s} \sum_{n \geq 1}\left(\tilde{\lambda}_{n}^{2} w_{0, n}^{2}+w_{1, n}^{2}\right)\left(\frac{(n+1) \pi}{L}\right)^{2 s}+\left|u_{1}^{(0)}(0)\right|^{2} \\
& \leq C\left(\sum_{n \geq 1}\left(\tilde{\lambda}_{n}^{2} w_{0, n}^{2}+w_{1, n}^{2}\right) \tilde{\lambda}_{n}^{2 s}+\left|u_{1}^{(0)}(0)\right|^{2}\right) \\
& \leq C\left(\left\|\left(w^{(0)}, w^{(1)}\right)\right\|_{D\left(\mathcal{A}_{D}^{s}\right)}^{2}+\left|u_{1}^{(0)}(0)\right|^{2}\right) .
\end{aligned}
$$

Consequently, by Lemma 3.7, we obtain that there exists $C>0$ such that

$$
E_{+}(0) \leq C\left\|\left(u^{(0)}, u^{(1)}\right)\right\|_{X_{s}}^{2} .
$$

Moreover,

$$
\sum_{n \geq 1} u_{n}=\frac{1}{2} \sum_{n \geq 1}\left(\tilde{\lambda}_{n}^{2} w_{0, n}^{2}+w_{1, n}^{2}\right)+u_{1}^{(0)}(0)^{2}=\left\|w^{(0)}\right\|_{\tilde{V}}^{2}+u_{1}^{(0)}(0)^{2}+\left\|u^{(1)}\right\|_{L^{2}(\mathcal{R})}^{2} .
$$

Furthermore, there exists $C^{\prime}>0$ such that

$$
\left\|u^{(0)}\right\|_{V}^{2}=\left\|w^{(0)}+u_{1}^{(0)}(0) \varphi\right\|_{V}^{2} \leq C^{\prime}\left(\left\|w^{(0)}\right\|_{V}^{2}+\left|u_{1}^{(0)}(0)\right|^{2}\right)=C^{\prime}\left(\left\|w^{(0)}\right\|_{\tilde{V}}^{2}+\left|u_{1}^{(0)}(0)\right|^{2}\right)
$$

because $\varphi$ is given and $w^{(0)} \in \tilde{V}$. Therefore, there exists $C^{\prime}>0$ such that

$$
\sum_{n \geq 1} u_{n} \geq C^{\prime}\left\|\left(u^{(0)}, u^{(1)}\right)\right\|_{V \times L^{2}(\mathcal{R})}^{2} .
$$

Consequently, (3.15) becomes, by the increasing character of $\Phi_{s}$

$$
1 \leq \Phi_{s}\left(\frac{E_{-}(0)}{C^{\prime}\left\|\left(u^{(0)}, u^{(1)}\right)\right\|_{V \times L^{2}(\mathcal{R})}^{2}}\right) \frac{C\left\|\left(u^{(0)}, u^{(1)}\right)\right\|_{X_{s}}^{2}}{C^{\prime}\left\|\left(u^{(0)}, u^{(1)}\right)\right\|_{V \times L^{2}(\mathcal{R})}^{2}},
$$

which yields

$$
E_{-}(0) \geq C^{\prime}\left\|\left(u^{(0)}, u^{(1)}\right)\right\|_{V \times L^{2}(\mathcal{R})}^{2} \Phi_{s}^{-1}\left(\frac{\left\|\left(u^{(0)}, u^{(1)}\right)\right\|_{V \times L^{2}(\mathcal{R})}^{2}}{C\left\|\left(u^{(0)}, u^{(1)}\right)\right\|_{X_{s}}^{2}}\right)
$$


with $C, C^{\prime}>0$. From (3.13) and (3.17), it follows that there exist $C, C^{\prime}>0$,

$$
E_{u}(T) \leq E_{u}(0)-C^{\prime}\left\|\left(u^{(0)}, u^{(1)}\right)\right\|_{V \times L^{2}(\mathcal{R})}^{2} \Phi_{s}^{-1}\left(\frac{\left\|\left(u^{(0)}, u^{(1)}\right)\right\|_{V \times L^{2}(\mathcal{R})}^{2}}{C\left\|\left(u^{(0)}, u^{(1)}\right)\right\|_{X_{s}}^{2}}\right),
$$

for any $\left(u^{(0)}, u^{(1)}\right) \in X_{s}$.

We follow now the proof of Ammari and Tucsnak [6]. We rewrite (3.18) as follows:

$$
\begin{aligned}
\left\|\left(u(T), u_{t}(T)\right)\right\|_{V \times L^{2}(\mathcal{R}) \leq}^{2} \leq & \left\|\left(u^{(0)}, u^{(1)}\right)\right\|_{V \times L^{2}(\mathcal{R})}^{2} \\
& -C^{\prime}\left\|\left(u^{(0)}, u^{(1)}\right)\right\|_{V \times L^{2}(\mathcal{R})}^{2} \Phi_{s}^{-1}\left(\frac{\left\|\left(u^{(0)}, u^{(1)}\right)\right\|_{V \times L^{2}(\mathcal{R})}^{2}}{C\left\|\left(u^{(0)}, u^{(1)}\right)\right\|_{X_{s}}^{2}}\right) .
\end{aligned}
$$

This estimate remains valid in successive time-intervals $[l T,(l+1) T]$. Notice that there exists $C>0$ such that

$$
\forall t \geq 0,\left\|\left(u(t), u_{t}(t)\right)\right\|_{X_{s}} \leq C\left\|\left(u^{(0)}, u^{(1)}\right)\right\|_{X_{s}},
$$

for $0<s<\frac{1}{2}$ by interpolation, because it is true for $s=0$ and $s=1$ (see Theorem 5.1 of [18]). By (3.19) and the fact that the energy is decreasing by (1.4) and that $\Phi_{s}^{-1}$ is increasing, we obtain that

$$
\begin{aligned}
& \left\|\left(u((l+1) T), u_{t}((l+1) T)\right)\right\|_{V \times L^{2}(\mathcal{R})}^{2} \leq\left\|\left(u(l T), u_{t}(l T)\right)\right\|_{V \times L^{2}(\mathcal{R})}^{2} \\
& -C^{\prime}\left\|\left(u(l T), u_{t}(l T)\right)\right\|_{V \times L^{2}(\mathcal{R})}^{2} \Phi_{s}^{-1}\left(\frac{\left\|\left(u((l+1) T), u_{t}((l+1) T)\right)\right\|_{V \times L^{2}(\mathcal{R})}^{2}}{C\left\|\left(u^{(0)}, u^{(1)}\right)\right\|_{X_{s}}^{2}}\right),
\end{aligned}
$$

for every $l \in \mathbb{N} \cup\{0\}$.

Our expression (3.20) coincides with (4.16) in Ammari and Tucsnak [6] (with $\mathcal{G}=\Phi_{s}^{-1}$ and $\theta=1 / 2$ ). The rest of the proof follows as in [6], where (3.4) is used, by noticing that

$$
\frac{\left\|\left(u((l+1) T), u_{t}((l+1) T)\right)\right\|_{V \times L^{2}(\mathcal{R})}^{2}}{C\left\|\left(u^{(0)}, u^{(1)}\right)\right\|_{X_{s}}^{2}}<1,
$$

taking a higher constant $C$ if necessary. Then (3.11) follows.

In addition, using (3.11) and making a particular and explicit choice of the concave function $\Phi_{s}$, we obtain a more explicit dependence of the weights on the decay rate of the energy:

THEOREM 3.10. Assume that the weighted observability inequality (2.1) holds for every solution of (1.7) with $\liminf _{n \rightarrow \infty} c_{n}=0$ and $c_{n} \neq 0$ for all $n \in \mathbb{N}^{*}$. Let $\omega$ be defined by (3.6). We set

$$
\forall t>0, \varphi(t)=\frac{\omega(t)}{t^{2}}
$$

Then there exists a constant $C>0$ such that for any initial data $\left(u^{(0)}, u^{(1)}\right) \in X_{s}(0<s<1 / 2)$, the corresponding solution $u$ of (1.1) verifies

$$
\forall t \geq 0, E_{u}(t) \leq \frac{C}{\left(\varphi^{-1}\left(\frac{1}{t+1}\right)\right)^{2 s}}\left\|\left(u^{(0)}, u^{(1)}\right)\right\|_{X_{s}}^{2} .
$$


Proof. We set

$$
\Phi_{s}(t)=\frac{1}{\left(\varphi^{-1}(t)\right)^{2 s}}
$$

where $0<s<1 / 2$. Then the pair $\left(\omega, \Phi_{s}\right)$ verifies $(3.1)-(3.2)$ on $(0, \infty)$. Indeed, as $\Phi_{s}$ is the composition of the functions $t \mapsto 1 / \varphi^{-1}(t)$ and $t \mapsto t^{2 s}$ which are increasing and concave functions (by Lemma 2.6 of [7]), $\Phi_{s}$ is an increasing and concave function.

Moreover, we easily check that

$$
\frac{1}{\varphi^{-1}(\omega(t))} \geq \frac{1}{t}
$$

on $[1, \infty)$. As a consequence, $(3.3)$ holds on $[1, \infty)$.

Finally, we have

$$
\frac{1}{t} \Phi_{s}^{-1}(t)=t^{\frac{1-s}{s}} \omega\left(\frac{1}{t^{\frac{1}{2 s}}}\right)
$$

which is an increasing function on $(0, \infty)$ because $0<s<1 / 2$, and therefore $\left(\omega, \Phi_{s}\right)$ satisfy (3.1)-(3.4). We apply now (3.11) of Theorem 3.8 with

$$
\Phi_{s}(t)=\left(\frac{1}{\varphi^{-1}(t)}\right)^{2 s},
$$

to obtain the result.

4. Examples. In [14] the authors proved observability inequalities of type (2.1) on which our analysis is based. In the case of star-shaped networks, the weights in (2.1) depend on the irrationality properties of the ratios of the lengths of the strings. In the case of tree-shaped networks, the observability inequality is proved under a condition (that is fulfilled generically within the class of tree-shaped networks) that generalizes the condition on the irrationality of the ratios of the lengths of the strings arising in the case of stars. Finally, in the case of general networks, the observability inequality holds under the condition that all eigenfunctions of the network are observable. This last result is a generalization of the previous one for stars and trees.

To illustrate the wide range of applications of the main result of this paper, in this section, we apply our previous results to some examples of particular networks: a star-shaped network and a particular tree. We obtain the weights $\left(c_{n}\right)_{n}$ directly by [14] and deduce an explicit decay rate for the corresponding dissipative system.

4.1. The star-shaped network with $N$ strings. The star-shaped network with $N$ strings is formed by $N$ strings connected at one point $v$, which constitutes a particular tree. Recall that the damping term is located on the vertex $v_{1}$, the origin of the controlled edge $e_{1}$ of length $l_{1}$. The remaining $N-1$ exterior nodes are denoted by $v_{i}, i=2, \ldots, N$, the string that contains $v_{i}$ by $e_{i}$ and its length by $l_{i}$.

In [14], the authors proved the observability inequality (2.1) for the conservative system (1.7) with Dirichlet boundary conditions at all exterior nodes with the following weights

$$
c_{k}=\max _{i=2, \ldots, N} \prod_{j \neq i}\left|\sin \left(\tilde{\lambda}_{k} l_{j}\right)\right|, \forall n \geq 1 .
$$




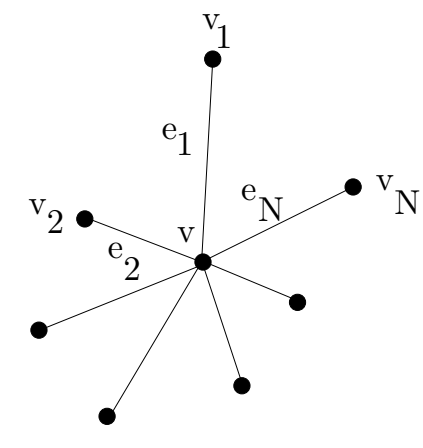

FIG. 4.1. A star-shaped network with $N$ strings

First, if the ratio of any two of the lengths of the uncontrolled strings is an irrational number, i.e. $l_{i} / l_{j} \notin \mathbb{Q}$ for all $i \neq j$, then for all $k \in \mathbb{N}$, we have $c_{k}>0$. In this case, the energy of $u$ solution of the dissipative system (1.1) tends to 0 as $t \rightarrow \infty$. This can be easily proved with LaSalle's invariance principle, using the energy as Lyapunov functional, but it does not yield any explicit decay rate.

Thus assume that $l_{i} / l_{j} \notin \mathbb{Q}$ for all $i \neq j$. Denote by $\mathcal{S}$ the set of all real numbers $\rho$ such that $\rho \notin \mathbb{Q}$ and so that its expansion as a continued fraction $\left[0, a_{1}, \ldots, a_{n}, \ldots\right]$ is such that $\left(a_{n}\right)$ is bounded. It is well known that $\mathcal{S}$ is uncountable and that its Lebesgue measure is zero. Roughly speaking, the set $\mathcal{S}$ contains all irrational numbers which are badly approximated by rational ones. In particular, by the Euler-Lagrange theorem, $\mathcal{S}$ contains all irrational quadratic numbers (i. e. the roots of second order equations with rational coefficients).

We use also a well-known result asserting that, for all $\epsilon>0$ there exists a set $B_{\epsilon} \subset \mathbb{R}$, such that the Lebesgue measure of $\mathbb{R} \backslash B_{\epsilon}$ is equal to zero, and a constant $C_{\epsilon}>0$ for which, if $\xi \in B_{\epsilon}$, then

$$
\|\xi m\| \geq \frac{C_{\epsilon}}{m^{1+\epsilon}}
$$

where \|\|$\eta \|$ is the distance from $\eta$ to the set $\mathbb{Z}$ :

$$
\left\||\eta \||=\min _{\eta-x \in \mathbb{Z}}|x| .\right.
$$

In particular, $\mathcal{S}$ is contained in the sets $B_{\epsilon}$ for every $\epsilon>0$ (see [14] for more details).

Then, by Corollary A.10 of [14], we have

Lemma 4.1. 1. If for all values $i, j=2, \ldots, N, i \neq j$, the ratios $l_{i} / l_{j}$ belong to $\mathcal{S}$, then there exists a constant $c>0$ such that

$$
c_{k} \geq \frac{c}{\tilde{\lambda}_{k}^{N-2}}, \forall k \in \mathbb{N}^{*}
$$

2. If for all values $i, j=2, \ldots, N, i \neq j$, the ratios $l_{i} / l_{j}$ belong to $B_{\epsilon}$, then there exists a 
constant $c_{\epsilon}>0$ such that

$$
c_{k} \geq \frac{c_{\epsilon}}{\tilde{\lambda}_{k}^{N-2+\epsilon}}, \forall k \in \mathbb{N}^{*}
$$

With more restrictive assumptions on the lengths of the uncontrolled strings, we have another bound for $c_{k}$. Let us recall a definition of [14].

Definition 4.2. We say that the real numbers $l_{1}, \ldots, l_{N}$ verify the conditions $(S)$ if

- $l_{1}, \ldots, l_{N}$ are linearly independent over the field $\mathbb{Q}$ of rational numbers;

- the ratios $l_{i} / l_{j}$ are algebraic numbers for $i, j=1, \ldots, N$. In this case, we have the following result (see Corollary A.10 of [14]):

LEMma 4.3. If the numbers $l_{2}, \ldots, l_{N}$ verify the conditions (S), then for every $\epsilon>0$, there exists a constant $c_{\epsilon}>0$ such that

$$
c_{k} \geq \frac{c_{\epsilon}}{\tilde{\lambda}_{k}^{1+\epsilon}}, \forall k \in \mathbb{N}^{*}
$$

Consequently, we have

Proposition 4.4. 1. Assume that for all values $i, j=2, \ldots, N, i \neq j$, the ratios $l_{i} / l_{j}$ belong to $\mathcal{S}$. Then there exists a constant $C>0$ such that for any initial data $\left(u^{(0)}, u^{(1)}\right) \in X_{s}(0<s<1 / 2)$, the corresponding solution $u$ of (1.1) verifies

$$
E_{u}(t) \leq \frac{C}{(t+1)^{\frac{s}{N-2}}}\left\|\left(u^{(0)}, u^{(1)}\right)\right\|_{X_{s}}^{2}
$$

2. Assume that for all values $i, j=2, \ldots, N, i \neq j$, the ratios $l_{i} / l_{j}$ belong to $B_{\epsilon}$ for $\epsilon>0$. Then there exists a constant $C_{\epsilon}>0$ such that for any initial data $\left(u^{(0)}, u^{(1)}\right) \in X_{s}(0<s<1 / 2)$, the corresponding solution $u$ of (1.1) verifies

$$
E_{u}(t) \leq \frac{C}{(t+1)^{\frac{s}{N-2+\epsilon}}}\left\|\left(u^{(0)}, u^{(1)}\right)\right\|_{X_{s}}^{2} .
$$

3. Assume that the numbers $l_{2}, \ldots, l_{N}$ verify the conditions $(S)$. Let $\epsilon>0$. Then there exists a constant $C_{\epsilon}>0$ such that for any initial data $\left(u^{(0)}, u^{(1)}\right) \in X_{s}(0<s<1 / 2)$, the corresponding solution $u$ of (1.1) verifies

$$
E_{u}(t) \leq \frac{C_{\epsilon}}{(t+1)^{\frac{s}{1+\epsilon}}}\left\|\left(u^{(0)}, u^{(1)}\right)\right\|_{X_{s}}^{2}
$$

Proof. We set $\alpha=N-2$ and $c_{\alpha}=c$ in the first case, $\alpha=N-2+\epsilon$ and $c_{\alpha}=c_{\epsilon}$ in the second one and $\alpha=1+\epsilon$ and $c_{\alpha}=c_{\epsilon}$ in the third one. For $\alpha \geq 1$, we take

$$
\omega(t)=d_{\alpha}^{2}\left(\frac{1}{t}\right)^{2 \alpha}
$$

where $d_{\alpha}$ verifies $d_{\alpha}^{2} \leq 1$ and $c_{\alpha}^{2} \tilde{\lambda}_{n-1}^{-2 \alpha} \geq d_{\alpha}^{2} n^{-2 \alpha}$ for all $n \in \mathbb{N}^{*}$, which is possible because of Weyl's formula (3.16).

We are in the situation 1 of Example 3.3 with $p=2 \alpha \geq 1$. Therefore, we take

$$
\Phi_{s}(t)=\left(\frac{t}{d_{\alpha}^{2}}\right)^{\frac{s}{\alpha}}
$$


Then $\left(\omega, \Phi_{s}\right)$ satisfy (3.1)-(3.4) and we apply Theorem 3.8 to finish the proof.

Finally, if the lengths $l_{2}, \ldots, l_{N}$ verify $l_{i} / l_{j} \notin \mathbb{Q}$ for all $i \neq j$, but the conditions 1,2 and 3 of Proposition 4.4 are not verified, it can be proved that $c_{n}$ verify

$$
c_{n} \geq \psi(n)>0, \forall n \in \mathbb{N}^{*},
$$

where $\psi$ is a positive convex and decreasing function which can be smaller than $c \tilde{\lambda}_{n}^{-\alpha}$, with $\alpha>0$. Indeed, we know by Appendix A of [14], that if we set

$$
a(\lambda)=\sum_{i=2}^{N} \prod_{j \neq i}\left|\sin \left(\lambda l_{j}\right)\right|,
$$

we have

$$
a(\lambda) \geq C \min _{i=2, \ldots, N} \prod_{j \neq i}\|\|_{l_{j}} m^{i}(\lambda) \|,
$$

where

$$
m^{i}(\lambda)=\mathbf{E}\left(\frac{l_{i}}{\pi} \lambda\right)
$$

$\mathbf{E}(\eta)$ being the closest integer number to $\eta:|\eta-\mathbf{E}(\eta)|=|\|\eta \mid\|$. Therefore, it is sufficient to bound by below $\left\|\left|\frac{l_{i}}{l_{j}} m\right|\right\|$ for all $m \in \mathbb{N}$ and $i \neq j$ to get a lower bound of $c_{n}$.

Moreover, we know that Liouville's numbers $\xi$ are such that for all $n \in \mathbb{N}$, there exists $q \in \mathbb{N}$, $q>1$ such that

$$
0<\||| \xi q\| \|<\frac{1}{q^{n+1}}
$$

That is why, when $l_{i} / l_{j}$ is a Liouville's number (see [8]), the function $\psi$ in (4.1) is smaller than any negative power of $\tilde{\lambda}_{n}$ (for example of the order of $e^{-n}$ ), and thus the decay rate of the energy of $u$ is slower than polynomial. Indeed, for instance, it is possible to construct real numbers $\xi$ of the form

$$
\xi=\sum_{k \in \mathbb{N}} 10^{-a_{k}}
$$

where $\left(a_{k}\right)_{k}$ is an increasing sequence of natural numbers, which are approximated by rational ones faster than any given positive increasing function $\rho$ :

$$
\| 10^{a_{p}} \xi|| \mid \rho\left(10^{a_{p}}\right)<\epsilon, \forall p \in \mathbb{N}
$$

for $\epsilon>0$ (see [14], p.66 for more details).

Let us construct an irrational number $x$ which is approximated by rational ones at an exponential speed. The aim is to find an irrational $x_{a}$ such that

$$
\liminf _{q \rightarrow \infty}\left\|\left|x_{a} q\right|\right\|^{\frac{1}{q}}=e^{-a} \text {, where } a \text { is a positive constant. }
$$


This construction uses the theory of continued fractions (see [21]). We recall here some results about the continued fractions which are used in the sequel and we refer to $[8,15,17]$ for more details. Let $\left[a_{0}, a_{1}, \ldots, a_{n}, \ldots\right]$ be the expansion as a continued fraction of $x \in \mathbb{R} \backslash \mathbb{Q}$. We set $x_{n}=\left[a_{0}, a_{1}, \ldots, a_{n}\right]=\frac{p_{n}}{q_{n}}$ where $p_{n}, q_{n}$ are integers. The integers $p_{n}, q_{n}$ are relatively prime and satisfy the following relations:

$$
p_{-1}=1, p_{0}=a_{0}, p_{n+1}=a_{n+1} p_{n}+p_{n-1}
$$

and

$$
q_{-1}=0, q_{0}=1, q_{n+1}=a_{n+1} q_{n}+q_{n-1} .
$$

First, we can notice that

$$
\liminf \||x q|\|^{\frac{1}{q}}=\liminf a_{n+1}^{-\frac{1}{q_{n}}}
$$

(see [21] for more details).

Thus finding $x_{a} \in \mathbb{R} \backslash \mathbb{Q}$ such that (4.2) is equivalent to finding $x_{a} \in \mathbb{R} \backslash \mathbb{Q}$ such that

$$
\liminf a_{n+1}^{-\frac{1}{q_{n}}}=e^{-a}
$$

We construct a such $x_{a}$ by induction. Set $a_{0}=0$, which determines $p_{0}$ and $q_{0}$. Then, if $a_{0}, \ldots, a_{n}$, $p_{0}, \ldots, p_{n}, q_{0}, \ldots, q_{n}$ are found, we choose $a_{n+1}$ by

$$
a_{n+1}=\mathbf{E}\left(e^{a q_{n}}\right) .
$$

Then, $p_{n+1}$ and $q_{n+1}$ are imposed by the relations (4.3) and (4.4).

Therefore, for all $a>0$, we have found an irrational number $x_{a}$ such that there exists a positive constant $\delta$ such that,

$$
\left\|\left|q x_{a}\right|\right\| \geq \delta e^{-a q}, \forall q \in \mathbb{N} .
$$

Consequently, if $l_{i} / l_{j}$ are reals of the form $x_{a_{i, j}}$ for $a_{i, j}>0$, which verify (4.6), then there exists a positive constant $C$ such that for all $k \in \mathbb{N}$,

$$
c_{k} \geq C e^{-b(N-2) \tilde{\lambda}_{k} \frac{l}{\pi}}
$$

where $b=\max _{i \neq j}\left(a_{i, j}\right)>0$ and $l=\max _{j}\left(l_{j}\right)$. By the Weyl's formula, there exists $c_{2}>0$ such that $\tilde{\lambda}_{k} \leq c_{2} k \pi / L$, and thus, we obtain

$$
c_{k}^{2} \geq C e^{-2 c_{2} b(N-2) k}=C e^{-A k},
$$

where $A=2 c_{2} b(N-2)$.

Consequently, we have

Proposition 4.5. Assume that for all values $i, j=2, \ldots, N, i \neq j$, the ratios $l_{i} / l_{j}$ are reals of the form $x_{a_{i, j}}$ for $a_{i, j}>0$ which verify (4.6). Then there exist constants $C, C^{\prime}>0$ such that for any initial data $\left(u^{(0)}, u^{(1)}\right) \in X_{s}(0<s<1 / 2)$, the corresponding solution $u$ of (1.1) verifies

$$
\forall t \geq 0, E_{u}(t) \leq \frac{C^{\prime}}{(\ln (C(1+t)))^{2 s}}\left\|\left(u^{(0)}, u^{(1)}\right)\right\|_{X_{s}}^{2} .
$$


Proof. We apply Theorem 3.8 with

$$
\omega(t)=C e^{-A t},
$$

for $C>0$ and $A>2(2 s+1)$, and

$$
\Phi_{s}(t)=\left(\frac{A}{\ln \left(\frac{C}{t}\right)}\right)^{2 s} .
$$

We are in the situation 2 of Example 3.3 and we simply apply (3.11) to obtain (4.7).

4.2. A non star-shaped tree. Now let us consider a tree, which is not star-shaped, having the simple structure in figure 4.2 .

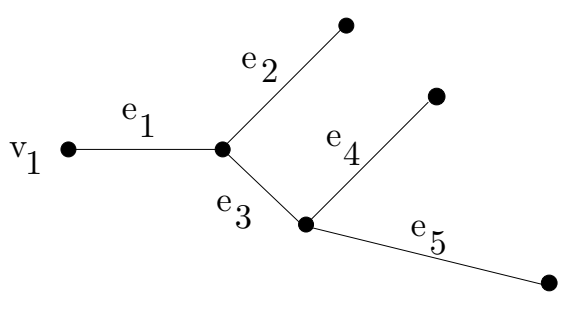

FIG. 4.2. A non star-shaped tree

Recall that the damping term is on the vertex $v_{1}$, the origin of the damped edge $e_{1}$ of length $l_{1}$. We will assume, in addition, that $l_{4}=l_{2}$.

Recall that in [14], the authors proved the observability inequality (2.1) for the conservative system (1.7) with Dirichlet boundary conditions at all exterior nodes with the weights $c_{k}$ given by

$$
c_{k}=\max \left\{\left|d_{5}\left(\tilde{\lambda}_{k}\right)\right|,\left|d_{4}\left(\tilde{\lambda}_{k}\right)\right|,\left|d_{2}\left(\tilde{\lambda}_{k}\right)\right|\right\},
$$

where

$$
\begin{gathered}
d_{5}(\lambda)=-\sin \left(\lambda l_{2}\right) \sin \left(\lambda l_{4}\right), d_{4}(\lambda)=-\sin \left(\lambda l_{2}\right) \sin \left(\lambda l_{5}\right) \\
d_{2}(\lambda)=-\left(\cos \left(\lambda l_{3}\right) \sin \left(\lambda l_{5}\right) \sin \left(\lambda l_{4}\right)+\sin \left(\lambda l_{3}\right) \cos \left(\lambda l_{5}\right) \sin \left(\lambda l_{4}\right)+\sin \left(\lambda l_{3}\right) \sin \left(\lambda l_{5}\right) \cos \left(\lambda l_{4}\right)\right) .
\end{gathered}
$$

First, if $c_{k}=0$, then $\left|d_{5}\left(\tilde{\lambda}_{k}\right)\right|=\left|d_{4}\left(\tilde{\lambda}_{k}\right)\right|=\left|d_{2}\left(\tilde{\lambda}_{k}\right)\right|=0$. As $l_{4}=l_{2}$, we obtain $\sin \left(\lambda l_{4}\right)=0$ and $\sin \left(\lambda l_{3}\right) \sin \left(\lambda l_{5}\right)=0$. If $\sin \left(\lambda l_{3}\right)=0$ (resp. $\left.\sin \left(\lambda l_{5}\right)=0\right)$, then, necessarily $l_{3} / l_{2}$ (resp. $\left.l_{5} / l_{2}\right)$ is a rational number. Consequently, $c_{k} \neq 0$ if $l_{3} / l_{2}$ and $l_{5} / l_{2}$ are irrational numbers and then, in this case, the energy of $u$ solution of (1.1) decays to 0 . 
Secondly, applying Appendix A of [14], we know that there exists a positive constant $c$ such that for every $k \in \mathbb{N}^{*}$,

$$
c_{k} \geq \frac{c}{\tilde{\lambda}_{k}^{\alpha}}
$$

if one of the following three conditions holds

- the ratios $l_{5} / l_{2}, l_{3} / l_{2}$ and $l_{3} / l_{5}$ belong to $\mathcal{S}$ and $\alpha>4$

or

- the ratios $l_{5} / l_{2}, l_{3} / l_{2}$ and $l_{3} / l_{5}$ belong to some $B_{\epsilon}$ and $\alpha>4+\epsilon$

or

- the numbers $l_{3}, l_{5}, l_{2}$ satisfy the conditions (S) and $\alpha>2+\epsilon$.

Consequently, by using (3.11) of Theorem 3.8 and the first statement of Example 3.3, there exists a constant $C>0$ such that for any initial data $\left(u^{(0)}, u^{(1)}\right) \in X_{s}(0<s<1 / 2)$, the corresponding solution $u$ verifies

$$
E_{u}(t) \leq \frac{C_{\epsilon}}{(t+1)^{\frac{s}{\alpha}}}\left\|\left(u^{(0)}, u^{(1)}\right)\right\|_{X_{s}}^{2} .
$$

Finally, if these previous conditions do not hold, we can apply Theorem 3.8 to obtain a decay rate of the energy of $u$ with an admissible pair $\left(\omega, \Phi_{s}\right)$, or Theorem 3.10 to obtain a more explicit decay rate of the energy.

As we have seen in these examples, our method to obtain decay rates of the dissipative system (1.1) is of systematic application:

First, we find the weights $\left(c_{n}^{2}\right)$ of the observability inequality (1.8) for the conservative problem (1.7) with Dirichlet boundary conditions at all exterior nodes.

Then we take, for the weight $\omega$, the lower convex envelop of the sequence $\left(1,\left(c_{n}^{2}\right)_{n}\right)$.

Finally we choose $\Phi_{s}$ such that $\left(\omega, \Phi_{s}\right)$ satisfy (3.1)-(3.4) to obtain the decay rate of the energy, given by

$$
\Phi_{s}\left(\frac{1}{1+t}\right) .
$$

Note that the study of the observability inequality (1.8) for (1.7) and the weights $\left(c_{n}^{2}\right)$ have been already done in some works (see [14]). Consequently we can use directly these results and it is not necessary to show another observability inequality. In addition, notice that the weighted observability inequality (2.7) holds under the same assumptions on the network that are needed for the Dirichlet problem (1.7). This method allows to treat an important class of networks.

Moreover we can extend this general principle to networks of strings with damping in several exterior vertices, or with damping in interior nodes.

Acknowledgement The first author would like thank Professor Serge Nicaise for fruitful discussions.

\section{REFERENCES}

[1] F. Ali Mehmeti. A characterization of a generalized $C^{\infty}$-notion on nets. Integral Equations Operator Theory, 9(6):753-766 (electronic), 1986.

[2] K. Ammari, A. Henrot, and M. Tucsnak. Asymptotic behaviour of the solutions and optimal location of the actuator for the pointwise stabilization of a string. Asymptot. Anal., 28(3-4):215-240, 2001. 
[3] K. Ammari and M. Jellouli. Stabilization of star-shaped networks of strings. Differential Integral Equations, 17(11-12):1395-1410, 2004.

[4] K. Ammari and M. Jellouli. Remark on stabilization of tree-shaped networks of strings. Appl. Math., 52(4):327343, 2007.

[5] K. Ammari, M. Jellouli, and M. Khenissi. Stabilization of generic trees of strings. J. Dyn. Control Syst., 11(2):177-193, 2005.

[6] K. Ammari and M. Tucsnak. Stabilization of second order evolution equations by a class of unbounded feedbacks. ESAIM Control Optim. Calc. Var., 6:361-386, 2001.

[7] P. Bégout and F. Soria. An interpolation inequality and its application to the stabilization of damped equations. J. Differ. Equations, 240(2):324-356, 2007.

[8] J. W. Cassals. An Introduction to Diophantine Approximation. Cambridge Univ. Press, Cambridge, UK, 1966.

[9] R. Dáger. Observation and control of vibrations in tree-shaped networks of strings. SIAM J. Control Optim., 43(2):590-623 (electronic), 2004.

[10] R. Dáger and E. Zuazua. Controllability of star-shaped networks of strings. In Mathematical and numerical aspects of wave propagation (Santiago de Compostela, 2000), pages 1006-1010. SIAM, Philadelphia, PA, 2000.

[11] R. Dáger and E. Zuazua. Controllability of star-shaped networks of strings. C. R. Acad. Sci. Paris Sér. I Math., 332(7):621-626, 2001.

[12] R. Dáger and E. Zuazua. Controllability of tree-shaped networks of vibrating strings. C. R. Acad. Sci. Paris Sér. I Math., 332(12):1087-1092, 2001.

[13] R. Dáger and E. Zuazua. Spectral boundary controllability of networks of strings. C. R. Math. Acad. Sci. Paris, 334(7):545-550, 2002.

[14] R. Dáger and E. Zuazua. Wave propagation, observation and control in 1-d flexible multi-structures, volume 50 of Mathématiques \&S Applications (Berlin) [Mathematics \& Applications]. Springer-Verlag, Berlin, 2006.

[15] G. H. Hardy and E. M. Wright. An introduction to the theory of numbers. The Clarendon Press Oxford University Press, New York, fifth edition, 1979.

[16] J. E. Lagnese, G. Leugering, and E. J. P. G. Schmidt. Modeling, analysis and control of dynamic elastic multi-link structures. Systems \& Control: Foundations \& Applications. Birkhäuser Boston Inc., Boston, MA, 1994.

[17] S. Lang. Introduction to Diophantine approximations. Addison-Wesley Publishing Co., Reading, Mass.London-Don Mills, Ont., 1966.

[18] J.-L. Lions and E. Magenes. Problèmes aux limites non homogènes et applications. Vol. 1. Travaux et Recherches Mathématiques, No. 17. Dunod, Paris, 1968.

[19] S. Nicaise. Spectre des réseaux topologiques finis. Bull. Sci. Math. (2), 111(4):401-413, 1987.

[20] S. Nicaise and J. Valein. Stabilization of the wave equation on 1-D networks with a delay term in the nodal feedbacks. Netw. Heterog. Media, 2(3):425-479 (electronic), 2007.

[21] H. Queffélec. Les nombres. Problèmes anciens et actuels. Mathémathèmes. Ellipses, 2000

[22] W. Rudin. Real and complex analysis. McGraw-Hill Book Co., New York, third edition, 1987.

[23] H. Triebel. Interpolation theory, function spaces, differential operators, volume 18 of North-Holland Mathematical Library. North-Holland Publishing Co., Amsterdam, 1978.

[24] J. von Below. A characteristic equation associated to an eigenvalue problem on $c^{2}$-networks. Linear Algebra Appl., 71:309-325, 1985.

[25] G. Q. Xu, D. Y. Liu, and Y. Q. Liu. Abstract second order hyperbolic system and applications to controlled networks of strings. SIAM J. Control Optim., 47(4):1762-1784 (electronic), 2008. 